\title{
Functional Encryption for Randomized Functionalities in the Private-Key Setting from Minimal Assumptions
}

\author{
Ilan Komargodski* Gil Segev ${ }^{\dagger} \quad$ Eylon Yogev*
}

\begin{abstract}
We present a construction of a private-key functional encryption scheme for any family of randomized functionalities based on any such scheme for deterministic functionalities that is sufficiently expressive. Instantiating our construction with existing schemes for deterministic functionalities, we obtain schemes for any family of randomized functionalities based on a variety of assumptions (including the LWE assumption, simple assumptions on multilinear maps, and even the existence of any one-way function) offering various trade-offs between security and efficiency.

Previously, Goyal, Jain, Koppula and Sahai [Cryptology ePrint Archive, 2013] constructed a public-key functional encryption scheme for any family of randomized functionalities based on indistinguishability obfuscation.

One of the key insights underlying our work is that, in the private-key setting, a sufficiently expressive functional encryption scheme may be appropriately utilized for implementing proof techniques that were so far implemented based on obfuscation assumptions (such as the punctured programming technique of Sahai and Waters [STOC 2014]). We view this as a contribution of independent interest that may be found useful in other settings as well.
\end{abstract}

${ }^{*}$ Department of Computer Science and Applied Mathematics, Weizmann Institute of Science, Rehovot 76100, Israel. Email: \{ilan.komargodski, eylon.yogev\}@weizmann.ac.il. Research supported in part by a grant from the Israel Science Foundation, the I-CORE Program of the Planning and Budgeting Committee, BSF and IMOS.

${ }^{\dagger}$ School of Computer Science and Engineering, Hebrew University of Jerusalem, Jerusalem 91904, Israel. Email: segev@cs.huji.ac.il. Supported by the European Union's Seventh Framework Programme (FP7) via a Marie Curie Career Integration Grant, by the Israel Science Foundation (Grant No. 483/13), and by the Israeli Centers of Research Excellence (I-CORE) Program (Center No. 4/11). 


\section{Introduction}

The cryptographic community's vision of functional encryption [SW08, BSW11, O'N10] is rapidly evolving. Whereas traditional encryption schemes offer an all-or-nothing guarantee when accessing encrypted data, functional encryption schemes offer tremendous flexibility. Specifically, such schemes support restricted decryption keys that allow users to learn specific functions of the encrypted data and nothing else.

Motivated by the early examples of functional encryption schemes for specific functionalities (such as identity-based encryption [Sha84, BF03, Coc01]), extensive research has recently been devoted to the construction of functional encryption schemes for rich and expressive families of functions (see, for example, [SW08, BSW11, O'N10, GVW12, AGV ${ }^{+} 13, \mathrm{BO} 13, \mathrm{BCP}_{14}, \mathrm{DIJ}^{+} 13$, $\mathrm{GGH}^{+} 13, \mathrm{GKP}^{+} 13$, Wat14, $\left.\mathrm{GGH}^{+} 14, \mathrm{BS} 14\right]$ and the references therein).

Until very recently, research on functional encryption has focused on the case of deterministic functions. More specifically, in a functional encryption scheme for a family $\mathcal{F}$ of deterministic functions, a trusted authority holds a master secret key msk that enables to generate a functional key $\mathrm{sk}_{f}$ for any function $f \in \mathcal{F}$. Now, anyone holding the functional key $\mathrm{sk}_{f}$ and an encryption of some value $x$, can compute $f(x)$ but cannot learn any additional information about $x$. In many scenarios, however, dealing only with deterministic functions may be insufficient, and a more general framework allowing randomized functions is required.

Functional encryption for randomized functionalities. Motivated by various real-world scenarios, Goyal et al. [GJK ${ }^{+} 13$ ] have recently put forward a generalization of functional encryption to randomized functionalities. In this setting, given a functional key $\mathrm{sk}_{f}$ for a randomized function $f$ and given an encryption of a value $x$, one should be able to obtain a sample from the distribution $f(x)$. As Goyal et al. pointed out, the case of randomized functions presents new challenges for functional encryption. These challenge arise already when formalizing the security of functional encryption for randomized functions ${ }^{1}$, and then become even more noticeable when designing such schemes.

Goyal et al. $\left[\mathrm{GJK}^{+} 13\right]$ presented a realistic framework for modeling the security of functional encryption schemes for randomized functionalities. Even more importantly, within their framework they constructed a public-key functional encryption scheme supporting the set of all randomized functionalities (that are computable by bounded-size circuits). Their construction builds upon the elegant approach of punctured programming due to Sahai and Waters [SW14], and they prove the security of their construction based on indistinguishability obfuscation [BGI ${ }^{+} 12, \mathrm{GGH}^{+} 13$ ].

Identifying the minimal assumptions for functional encryption. The work of Goyal et al. $\left[\mathrm{GJK}^{+} 13\right]$ naturally gives rise to the intriguing question of whether functional encryption for randomized functionalities can be based on assumptions that are seemingly weaker than indistinguishability obfuscation. On one hand, it may be the case that functional encryption for randomized functionalities is indeed a significantly more challenging primitive than functional encryption for deterministic functionalities. In this case, it would be conceivable to use the full power of indistinguishability obfuscation for constructing such schemes. On the other hand, however, it may be possible that a functional encryption scheme for randomized functions can be constructed in a direct black-box manner from any such scheme for deterministic functions.

\footnotetext{
${ }^{1}$ For example, an adversary holding a functional key $\mathrm{sk}_{f}$ and an encryption of a value $x$, should not be able to tamper with the randomness that is used for sampling from distribution $f(x)$. This is extremely well motivated by the examples provided by Goyal et al. in the contexts of auditing an encrypted database via randomized sampling, and of performing differentially-private analysis on an encrypted database via randomized perturbations. We refer the reader to $\left[\mathrm{GJK}^{+} 13\right]$ for more details.
} 
This question is especially interesting since various functional encryption schemes for (general) deterministic functionalities are already known to exist based on assumptions that seem significantly weaker than indistinguishability obfuscation (such as Learning with Errors assumption or even the existence of any one-way function) offering various trade-offs between security and efficiency (see Section 2.2 for more details on the existing schemes).

\subsection{Our Contributions}

In this work we consider functional encryption in the private-key setting, where the master secret key is used both for generating functional keys and for encryption. In this setting we provide an answer to the above question: we present a construction of a private-key functional encryption scheme for any family $\mathcal{F}$ of randomized functions based on any private-key functional encryption scheme for deterministic functions that is sufficiently expressive ${ }^{2}$. Inspired by the work of Goyal et al. [GJK ${ }^{+} 13$ ] in the public-key setting, we prove the security of our construction within a similarly well-motivated framework for capturing the security of private-key functional encryption for randomized functions.

Instantiations. Our resulting scheme inherits the flavor of security guaranteed by the underlying scheme (e.g., full vs. selective security, and one-key vs. many-keys security), and can be instantiated by a variety of existing functional encryption schemes. Specifically, our scheme can be based either on the Learning with Errors assumption, on obfuscation assumptions, on multilinear-maps assumptions, or even on the existence of any one-way function (offering various trade-offs between security and efficiency - we refer the reader to Section 2.2 for more details on the possible instantiations).

Applicable scenarios. Following-up on the motivating applications given by Goyal et al. [GJK ${ }^{+} 13$ ] in the contexts of auditing an encrypted database via randomized sampling, and of performing differentially-private analysis on an encrypted database via randomized perturbations, we observe that these two examples are clearly valid in the private-key setting as well. Specifically, in both applications, the party that provides functional keys is more than likely the same one who encrypts the data.

Obfuscation-based techniques via function privacy. One of the key insights underlying our work is that in the private-key setting, where encryption is performed honestly by the owner of the master secret key, the power of indistinguishability obfuscation may not be needed. Specifically, we observe that in some cases one can instead rely on the weaker notion of function privacy [SSW09, BRS13a, $\mathrm{AAB}^{+}$13, BS14]. Intuitively, a functional encryption scheme is function private if a functional key $\mathrm{sk}_{f}$ for a function $f$ reveals no "unnecessary" information on $f$. For functional encryption in the private-key setting, this essentially means that encryptions of messages $m_{1}, \ldots, m_{T}$ together with functional keys corresponding to functions $f_{1}, \ldots, f_{T}$ reveal essentially no information other than the values $\left\{f_{i}\left(m_{j}\right)\right\}_{i, j \in[T]}$. Brakerski and Segev [BS14] recently showed that a functionprivate scheme can be obtained from any private-key functional encryption scheme.

Building upon the notion of function privacy, we show that any private-key functional encryption scheme may be appropriately utilized for implementing some of the proof techniques that were so far implemented based on indistinguishability obfuscation. These include, in particular, a variant of the punctured programming approach of Sahai and Waters [SW14]. We view this as a contribution of independent interest that may be found useful in other settings as well.

\footnotetext{
${ }^{2}$ Our only assumption on the underlying scheme is that it supports the family $\mathcal{F}$ (when viewed as a family of singleinput deterministic functions), supports the evaluation procedure of a pseudorandom function family, and supports a few additional basic operations (such as conditional statements).
} 


\subsection{Additional Related Work}

A related generalization of functional encryption is that of functional encryption for multiple-input functions due to Goldwasser et al. $\left[\mathrm{GGG}^{+} 14\right]$. A multiple-input functional encryption scheme for a function family $\mathcal{F}$ allows generating a functional key $\mathrm{sk}_{f}$ for any function $f \in \mathcal{F}$, and this enables to compute $f(x, y)$ given an encryption of $x$ and an encryption of $y$, while not learning any additional information. Although capturing the security guarantees that can be provided by such schemes is quite challenging, multiple-input functional encryption might be useful for dealing with single-input randomized functionalities: One can view a randomized function $f(x ; r)$ as a two-input function, where its first input is the actual input $x$, and its second input is the randomness $r$ (that is possibly derived by a PRF key). However, the construction of Goldwasser et al. is based on indistinguishability obfuscation, and our goal is to rely on weaker assumptions. In addition, it is not clear that the notion of security of Goldwasser et al. suffices for capturing our notion of "best-possible" message privacy which allows for an a-priori non-negligible advantage in distinguishing the output distributions of two randomized functions (see Sections 1.3 and 3 for our notion of privacy).

Our construction relies on the notion of function privacy for functional encryption schemes, first introduced by Boneh et al. [BRS13a, BRS13b] in the public-key setting, and then studied by Agrawal et al. $\left[\mathrm{AAB}^{+} 13\right]$ and by Brakerski and Segev [BS14] in the private-key setting (generalizing the work on predicate privacy in the private-key setting by Shen et al. [SSW09]). As discussed in Section 1.1, for functional encryption in the private-key setting, function privacy essentially means that encryptions of messages $m_{1}, \ldots, m_{T}$ together with functional keys corresponding to functions $f_{1}, \ldots, f_{T}$ reveal essentially no information other than the values $\left\{f_{i}\left(m_{j}\right)\right\}_{i, j \in[T]}$. In terms of underlying assumptions, we rely on the fact that Brakerski and Segev [BS14] showed that a function-private scheme can be obtained from any private-key functional encryption scheme.

\subsection{Overview of Our Approach}

A private-key functional encryption scheme for a family $\mathcal{F}$ of randomized functions consists of four probabilistic polynomial-time algorithms (Setup, KG, Enc, Dec). The syntax is identical to that of functional encryption for deterministic functions (see Section 2.2), but the correctness and security requirements are more subtle. In this section we begin with a brief overview of our notions of correctness and security. Then, we provide a high-level overview of our new construction, and the main ideas and challenges underlying its proof of security.

Correctness and independence of decrypted values. Our notion of correctness follows that of Goyal et al. [GJK $\left.{ }^{+} 13\right]$ by adapting it to the private-key setting. Specifically, we ask that for any sequence of messages $x_{1}, \ldots, x_{T}$ and for any sequence of functions $f_{1}, \ldots, f_{T} \in \mathcal{F}$, it holds that the distribution obtained by encrypting $x_{1}, \ldots, x_{T}$ and then decrypting the resulting ciphertexts with functional keys corresponding to $f_{1}, \ldots, f_{T}$ is computationally indistinguishable from the distribution $\left\{f_{j}\left(x_{i} ; r_{i, j}\right)\right\}_{i, j \in[T]}$ where the $r_{i, j}$ 's are sampled independently and uniformly at random. As noted by Goyal et al. [GJK $\left.{ }^{+} 13\right]$, unlike in the case of deterministic functions where is suffices to define correctness for a single ciphertext and a single key, here it is essential to define correctness for multiple (possibly correlated) ciphertexts and keys. We refer the reader to Section 3.1 for our formal definition.

"Best-possible" message privacy. As in functional encryption for deterministic functions, we consider adversaries whose goal is to distinguish between encryptions of two challenge messages, $x_{0}^{*}$ and $x_{1}^{*}$, when given access to an encryption oracle (as required in private-key encryption) and to functional keys of various functions. Recall that in the case of deterministic functions, the adversary is allowed to ask for functional keys for any function $f$ such that $f\left(x_{0}^{*}\right)=f\left(x_{1}^{*}\right)$. 
When dealing with randomized functions, however, it is significantly less clear how to prevent adversaries from choosing functions $f$ that will enable to easily distinguish between encryptions of $x_{0}^{*}$ and $x_{1}^{*}$. Our notions of message privacy ask that the functional encryption scheme under consideration will not add a non-negligible advantage to the (possibly non-negligible) advantage that adversaries may already have in distinguishing between the distributions $f\left(x_{0}^{*}\right)$ and $f\left(x_{1}^{*}\right)$. That is, given that adversaries are able to obtain a sample from the distribution $f\left(x_{0}^{*}\right)$ or from the distribution $f\left(x_{1}^{*}\right)$ using the functional key $\mathbf{s k}_{f}$, and may already have some advantage in distinguishing these distributions, we ask for "best-possible" message privacy in the sense that essentially no additional advantage can be gained.

Concretely, if the distributions $f\left(x_{0}^{*}\right)$ and $f\left(x_{1}^{*}\right)$ can be efficiently distinguished with advantage at most $\Delta=\Delta(\lambda)$ to begin with (where $\Delta$ does not necessarily have to be negligible), then we require that no adversary that is given a functional key for $f$ will be able to distinguish between encryptions of $x_{0}^{*}$ and $x_{1}^{*}$ with advantage larger than $\Delta+\operatorname{neg}(\lambda)$, for some negligible function neg $(\cdot)$. More generally, an adversary that is given functional keys for $T=T(\lambda)$ such functions (and access to an encryption oracle), should not be able to distinguish between encryptions of $x_{0}^{*}$ and $x_{1}^{*}$ with advantage larger than $T \cdot \Delta+\operatorname{neg}(\lambda)$. We note that our approach for realistically capturing message privacy somewhat differs from that of Goyal et al. [GJK $\left.{ }^{+} 13\right]$, and we refer the reader to Appendix A for a brief comparison between the two approaches ${ }^{3}$.

We put forward two flavors of "best-possible" message privacy, a non-adaptive flavor and an adaptive flavor, depending on the flavor of indistinguishability guarantee that is satisfied by the function family under consideration. We refer the reader to Section 3.2 for our formal definitions, and for some typical examples of function families that satisfy each flavor.

Our construction. Let (Setup, KG, Enc, Dec) be any private-key functional encryption scheme that provides message privacy and function privacy ${ }^{4}$. Our new scheme is quite intuitive and is described as follows:

- The setup and decryption algorithms are identical to those of the underlying scheme.

- The encryption algorithm on input a message $x$, samples a string $s$ uniformly at random, and outputs an encryption ct $\leftarrow \operatorname{Enc}(\operatorname{msk},(x, \perp, s, \perp))$ of $x$ and $s$ together with two additional "empty slots" that will be used in the security proof.

- The key-generation algorithm on input a description of a randomized function $f$, samples a PRF key $K$, and outputs a functional key for the deterministic function Left $_{f, K}$ defined as follows: On input $\left(x_{L}, x_{R}, s, z\right)$ output $f\left(x_{L} ; r\right)$ where $r=\operatorname{PRF}_{K}(s)$.

The correctness and independence of our scheme follow in a straightforward manner from the correctness of the underlying scheme and the assumption that PRF is pseudorandom. In fact, it suffices that PRF is weakly pseudorandom (i.e., computationally indistinguishable from a truly random function when evaluated on independent and uniformly sampled inputs).

As for the message privacy of the scheme, recall that we consider adversaries that can access an encryption oracle and a key-generation oracle, and should not be able to distinguish between an encryption Enc $\left(\mathrm{msk},\left(x_{0}^{*}, \perp, s^{*}, \perp\right)\right)$ of $x_{0}^{*}$ and an encryption $\operatorname{Enc}\left(\mathrm{msk},\left(x_{1}^{*}, \perp, s^{*}, \perp\right)\right)$ of $x_{1}^{*}$ with advantage larger than $T \cdot \Delta+\operatorname{neg}(\lambda)$ (where $T$ is the number of functional keys given to the

\footnotetext{
${ }^{3}$ As far as we are able to currently tell, it seems that both our scheme and the scheme of Goyal et al. [GJK ${ }^{+} 13$ ] provide message privacy according to both of these approaches. We emphasize that we view the main contribution of our paper as basing the security of our scheme on any underlying functional encryption scheme (and avoiding obfuscation-related assumptions), and not as offering alternative notions of message privacy.

${ }^{4}$ As discussed above, function privacy can be assumed without loss of generality using the transformation of Brakerski and Segev [BS14].
} 
adversary, and $\Delta$ is the a-priori distinguishing advantage for the functions under consideration as described above).

The first step in our proof of security is to replace the challenge ciphertext with a modified challenge ciphertext $\operatorname{Enc}\left(\mathrm{msk},\left(x_{0}^{*}, x_{1}^{*}, s^{*}, \perp\right)\right)$ that contains information on both challenge messages (this is made possible due to the message privacy of the underlying scheme). Next, denoting the adversary's key-generation queries by $f_{1}, \ldots, f_{T}$, our goal is to replace the functional keys Left $f_{1}, K_{1}, \ldots$, Left $f_{T}, K_{T}$ with the functional keys Right $_{f_{1}, K_{1}}, \ldots$, Right $f_{T}, K_{T}$, where the function Right $_{f, K}$ is defined as follows: On input $\left(x_{L}, x_{R}, s, z\right)$ output $f\left(x_{R} ; r\right)$ where $r=\operatorname{PRF}_{K}(s)$. At this point we note that, from the adversary's point of view, when providing only Left keys the modified challenge ciphertext is indistinguishable from an encryption of $x_{0}^{*}$, and when providing only Right keys the modified challenge ciphertext is indistinguishable from an encryption of $x_{1}^{*}$.

The most challenging part of the proof is in bounding the adversary's advantage in distinguishing the sequences of Left and Right keys, based on the function privacy and the message privacy of the underlying scheme. The basic idea is to switch the functional keys from Left to Right one by one, following different proof strategies for pre-challenge keys and for post-challenge keys ${ }^{5}$.

When dealing with a pre-challenge key $\mathrm{sk}_{f}$, the function $f$ is already known when producing the challenge ciphertext. Therefore, we can use the message privacy of the underlying scheme and replace the (already-modified) challenge ciphertext with $\operatorname{Enc}\left(\mathrm{msk},\left(x_{0}^{*}, x_{1}^{*}, s^{*}, z^{*}\right)\right)$, where $z^{*}=f\left(x_{0}^{*} ; r^{*}\right)$ and $r^{*}=\operatorname{PRF}_{K}\left(s^{*}\right)$. Then, we use the function privacy of the underlying scheme, and replace the functional key Left $f_{, K}$ with a functional key for the function OutputZ that simply outputs $z$ whenever $s=s^{*}$. From this point on, we use the pseudorandomness of PRF and replace $r^{*}=\operatorname{PRF}_{K}\left(s^{*}\right)$ with a truly uniform $r^{*}$, and then replace $z^{*} \leftarrow f\left(x_{0}^{*}\right)$ with $z^{*} \leftarrow f\left(x_{1}^{*}\right)$. Similar steps then enable us to replace the functional key OutputZ with a functional key for the function Right ${ }_{f, K}$.

When dealing with a post-challenge key $\mathrm{sk}_{f}$, we would like to follow the same approach of embedding the value $f\left(x_{0}^{*} ; r^{*}\right)$ or $f\left(x_{1}^{*} ; r^{*}\right)$. However, for post-challenge keys, the function $f$ is not known when producing the challenge ciphertext. Instead, in this case, the challenge messages $x_{0}^{*}$ and $x_{1}^{*}$ are known when producing the functional key $\mathrm{sk}_{f}$. Combining this with the function privacy of the underlying scheme enables us to embed the above values in the functional key $\mathrm{sk}_{f}$, and once again replace the Left keys with the Right keys. We refer the reader to Section 4 for the formal description of our scheme and its proof of security.

\subsection{Paper Organization}

The remainder of this paper is organized as follows. In Section 2 we provide an overview of the basic notation and standard tools underlying our construction. In Section 3 we introduce our notions of security for private-key functional encryption schemes for randomized functionalities. In Section 4 we present our new scheme and prove its security. Formal proofs of the claims that are stated in Section 4 appear in Appendices B and C.

\section{Preliminaries}

In this section we present the notation and basic definitions that are used in this work. For a distribution $X$ we denote by $x \leftarrow X$ the process of sampling a value $x$ from the distribution $X$. Similarly, for a set $\mathcal{X}$ we denote by $x \leftarrow \mathcal{X}$ the process of sampling a value $x$ from the uniform distribution over $\mathcal{X}$. For a randomized function $f$ and an input $x \in \mathcal{X}$, we denote by $y \leftarrow f(x)$ the process of sampling a value $y$ from the distribution $f(x)$. For an integer $n \in \mathbb{N}$ we denote by $[n]$

\footnotetext{
${ }^{5}$ We use the term pre-challenge keys for all functional keys that are obtained before the challenge phase, and the term post-challenge keys for all functional keys that are obtained after the challenge phase.
} 
the set $\{1, \ldots, n\}$. A function neg $: \mathbb{N} \rightarrow \mathbb{R}$ is negligible if for every constant $c>0$ there exists an integer $N_{c}$ such that $\operatorname{neg}(\lambda)<\lambda^{-c}$ for all $\lambda>N_{c}$.

The statistical distance between two random variables $X$ and $Y$ over a finite domain $\Omega$ is defined as $\operatorname{SD}(X, Y)=\frac{1}{2} \sum_{\omega \in \Omega}|\operatorname{Pr}[X=\omega]-\operatorname{Pr}[Y=\omega]|$. Two sequences of random variables $X=\left\{X_{\lambda}\right\}_{\lambda \in \mathbb{N}}$ and $Y=\left\{Y_{\lambda}\right\}_{\lambda \in \mathbb{N}}$ are computationally indistinguishable if for any probabilistic polynomial-time algo$\operatorname{rithm} \mathcal{A}$ there exists a negligible function neg $(\cdot)$ such that $\left|\operatorname{Pr}\left[\mathcal{A}\left(1^{\lambda}, X_{\lambda}\right)=1\right]-\operatorname{Pr}\left[\mathcal{A}\left(1^{\lambda}, Y_{\lambda}\right)=1\right]\right| \leq$ $\operatorname{neg}(\lambda)$ for all sufficiently large $\lambda \in \mathbb{N}$.

\subsection{Pseudorandom Functions}

Let $\left\{\mathcal{K}_{\lambda}, \mathcal{X}_{\lambda}, \mathcal{Y}_{\lambda}\right\}_{\lambda \in \mathbb{N}}$ be a sequence of sets and let PRF $=$ (PRF.Gen, PRF.Eval) be a function family with the following syntax:

- PRF.Gen is a probabilistic polynomial-time algorithm that takes as input the unary representation of the security parameter $\lambda$, and outputs a key $K \in \mathcal{K}_{\lambda}$.

- PRF.Eval is a deterministic polynomial-time algorithm that takes as input a key $K \in \mathcal{K}_{\lambda}$ and a value $x \in \mathcal{X}_{\lambda}$, and outputs a value $y \in \mathcal{Y}_{\lambda}$.

The sets $\mathcal{K}_{\lambda}, \mathcal{X}_{\lambda}$, and $\mathcal{Y}_{\lambda}$ are referred to as the key space, domain, and range of the function family, respectively. For easy of notation we may denote by PRF.Eval $K(\cdot)$ or $\operatorname{PRF}_{K}(\cdot)$ the function PRF.Eval $(K, \cdot)$ for $K \in \mathcal{K}_{\lambda}$. The following is the standard definition of a pseudorandom function family.

Definition 2.1 (Pseudorandomness). A function family PRF $=$ (PRF.Gen, PRF.Eval) is pseudorandom if for every probabilistic polynomial-time algorithm $\mathcal{A}$ there exits a negligible function $n e g(\cdot)$ such that

$$
\operatorname{Adv}_{\mathrm{PRF}, \mathcal{A}}(\lambda) \stackrel{\text { def }}{=}\left|\underset{K \leftarrow \operatorname{PRF} . \operatorname{Gen}\left(1^{\lambda}\right)}{\operatorname{Pr}}\left[\mathcal{A}^{\mathrm{PRF} . \operatorname{Eval}_{K}(\cdot)}\left(1^{\lambda}\right)=1\right]-\operatorname{Pr}_{f \leftarrow F_{\lambda}}\left[\mathcal{A}^{f(\cdot)}\left(1^{\lambda}\right)=1\right]\right| \leq \operatorname{neg}(\lambda),
$$

for all sufficiently large $\lambda \in \mathbb{N}$, where $F_{\lambda}$ is the set of functions that map $\mathcal{X}_{\lambda}$ into $\mathcal{Y}_{\lambda}$.

In addition to the standard notion of a pseudorandom function family, we rely on the seemingly stronger (yet existentially equivalent) notion of a puncturable pseudorandom function family $\left[\mathrm{KPT}^{+} 13, \mathrm{BW} 13, \mathrm{SW} 14, \mathrm{BGI14}\right]$. In terms of syntax, this notion asks for an additional probabilistic polynomial-time algorithm, PRF.Punc, that takes as input a key $K \in \mathcal{K}_{\lambda}$ and a set $S \subseteq \mathcal{X}_{\lambda}$ and outputs a "punctured" key $K_{S}$. The properties required by such a puncturing algorithm are capture by the following definition.

Definition 2.2 (Puncturable PRF). A pseudorandom function family PRF = (PRF.Gen, PRF.Eval, PRF.Punc) is puncturable if the following properties are satisfied:

1. Functionality: For all sufficiently large $\lambda \in \mathbb{N}$, for every set $S \subseteq \mathcal{X}_{\lambda}$, and for every $x \in \mathcal{X}_{\lambda} \backslash S$ it holds that

$$
\left.\underset{\substack{K \leftarrow \text { PRF.Gen }\left(1^{\lambda}\right) ; \\ K_{S} \leftarrow \operatorname{PRF} . \operatorname{Punc}(K, S)}}{\text { PRF.Eval }_{K}(x)=\text { PRF.Eval }} K_{S}(x)\right]=1 .
$$

2. Pseudorandomness at Punctured Points: Let $\mathcal{A}=\left(\mathcal{A}_{1}, \mathcal{A}_{2}\right)$ be any probabilistic polyomial-time algorithm such that $\mathcal{A}_{1}\left(1^{\lambda}\right)$ outputs a set $S \subseteq \mathcal{X}_{\lambda}$, a value $x \in S$, and state information state. Then, for any such $\mathcal{A}$ there exists a negligible function neg(·) such that

$$
\operatorname{Adv}_{\text {puPRF }, \mathcal{A}}(\lambda) \stackrel{\text { def }}{=} \mid \operatorname{Pr}\left[\mathcal{A}_{2}\left(K_{S}, \operatorname{PRF} . E \operatorname{Eval}_{K}(x), \text { state }\right)=1\right]-\operatorname{Pr}\left[\mathcal{A}_{2}\left(K_{S}, y, \text { state }\right)=1\right] \mid \leq \operatorname{neg}(\lambda)
$$


for all sufficiently large $\lambda \in \mathbb{N}$, where $(S, x$, state $) \leftarrow \mathcal{A}_{1}\left(1^{\lambda}\right), K \leftarrow \operatorname{PRF} . G e n\left(1^{\lambda}\right), K_{S}=$ $\operatorname{PRF} . \operatorname{Punc}(K, S)$, and $y \leftarrow \mathcal{Y}_{\lambda}$.

As observed by $\left[\mathrm{KPT}^{+} 13\right.$, BW13, SW14, BGI14] the GGM construction [GGM86] of PRFs from one-way functions can be easily altered to yield a puncturable PRF.

\section{$2.2 \quad$ Private-Key Functional Encryption}

A private-key functional encryption scheme over a message space $\mathcal{X}=\left\{\mathcal{X}_{\lambda}\right\}_{\lambda \in \mathbb{N}}$ and a function space $\mathcal{F}=\left\{\mathcal{F}_{\lambda}\right\}_{\lambda \in \mathbb{N}}$ is a quadruple (Setup, KG, Enc, Dec) of probabilistic polynomial-time algorithms. The setup algorithm Setup takes as input the unary representation $1^{\lambda}$ of the security parameter $\lambda \in \mathbb{N}$ and outputs a master-secret key msk. The key-generation algorithm KG takes as input a mastersecret key msk and a function $f \in \mathcal{F}_{\lambda}$, and outputs a functional key $\mathrm{sk}_{f}$. The encryption algorithm Enc takes as input a master-secret key msk and a message $x \in \mathcal{X}_{\lambda}$, and outputs a ciphertext ct. In terms of correctness we require that for all sufficiently large $\lambda \in \mathbb{N}$, for every function $f \in \mathcal{F}_{\lambda}$ and message $x \in \mathcal{X}_{\lambda}$ it holds that $\operatorname{Dec}(\mathrm{KG}($ msk, $f)$, Enc(msk, $\left.x)\right)=f(x)$ with all but a negligible probability over the internal randomness of the algorithms Setup, KG, and Enc.

In terms of security, we rely on the private-key variants existing indistinguishability-based notions for message privacy (see, for example, [BSW11, O’N10, BO13]) and function privacy (see $\left[\mathrm{AAB}^{+} 13\right.$, BS14]). When formalizing these notions it would be convenient to use the following standard notion of a left-or-right oracle.

Definition 2.3 (Left-or-right oracle). Let $\mathcal{O}(\cdot, \cdot)$ be a probabilistic two-input functionality. For each $b \in\{0,1\}$ we denote by $\mathcal{O}_{b}$ the probabilistic three-input functionality $\mathcal{O}_{b}\left(k, z_{0}, z_{1}\right) \stackrel{\text { def }}{=} \mathcal{O}\left(k, z_{b}\right)$.

\subsubsection{Message Privacy}

A functional encryption scheme is message private if the encryptions of any two messages $x_{0}$ and $x_{1}$ are computationally indistinguishable given access to an encryption oracle (as required in privatekey encryption) and to functional keys for any function $f$ such that $f\left(x_{0}^{*}\right)=f\left(x_{1}^{*}\right)$. We consider two variants of message privacy: ( full) message privacy in which adversaries are fully adaptive, and selective-function message privacy in which adversaries must issue their key-generation queries in advance.

Definition 2.4 (Message privacy). A functional encryption scheme FE = (Setup, KG, Enc, Dec) over a message space $\mathcal{X}=\left\{\mathcal{X}_{\lambda}\right\}_{\lambda \in \mathbb{N}}$ and a function space $\mathcal{F}=\left\{\mathcal{F}_{\lambda}\right\}_{\lambda \in \mathbb{N}}$ is message private if for any probabilistic polynomial-time adversary $\mathcal{A}$ there exists a negligible function neg $(\cdot)$ such that

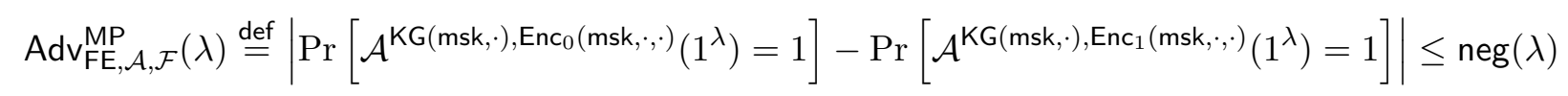

for all sufficiently large $\lambda \in \mathbb{N}$, where for every $\left(x_{0}, x_{1}\right) \in \mathcal{X}_{\lambda} \times \mathcal{X}_{\lambda}$ and $f \in \mathcal{F}_{\lambda}$ with which $\mathcal{A}$ queries the oracles $\mathrm{Enc}_{b}$ and $\mathrm{KG}$, respectively, it holds that $f\left(x_{0}\right)=f\left(x_{1}\right)$. Moreover, the probability is taken over the choice of msk $\leftarrow \operatorname{Setup}\left(1^{\lambda}\right)$ and the internal randomness of $\mathcal{A}$.

Definition 2.5 (Selective-function message privacy). A functional encryption scheme FE = (Setup, KG, Enc, Dec) over a message space $\mathcal{X}=\left\{\mathcal{X}_{\lambda}\right\}_{\lambda \in \mathbb{N}}$ and a function space $\mathcal{F}=\left\{\mathcal{F}_{\lambda}\right\}_{\lambda \in \mathbb{N}}$ is $T$-selectivefunction message private, where $T=T(\lambda)$, if for any probabilistic polynomial-time adversary $\mathcal{A}=$ $\left(\mathcal{A}_{1}, \mathcal{A}_{2}\right)$ there exists a negligible function neg $(\cdot)$ such that

$$
\operatorname{Adv}_{\mathrm{FE}, \mathcal{A}, \mathcal{F}, T}^{\mathrm{sfMP}}(\lambda) \stackrel{\text { def }}{=}\left|\operatorname{Pr}\left[\operatorname{Expt}_{\mathrm{FE}, \mathcal{A}, \mathcal{F}, T}^{(0)}(\lambda)=1\right]-\operatorname{Pr}\left[\operatorname{Expt}_{\mathrm{FE}, \mathcal{A}, \mathcal{F}, T}^{(1)}(\lambda)=1\right]\right| \leq \operatorname{neg}(\lambda)
$$


for all sufficiently large $\lambda \in \mathbb{N}$, where for each $b \in\{0,1\}$ and $\lambda \in \mathbb{N}$ the random variable $\operatorname{Expt}_{\mathrm{FE}, \mathcal{A}, \mathcal{F}, T}^{(b)}(\lambda)$ is defined as follows:

1. $\mathrm{msk} \leftarrow \operatorname{Setup}\left(1^{\lambda}\right)$.

2. $\left(f_{1}, \ldots, f_{T}\right.$, state $) \leftarrow \mathcal{A}_{1}\left(1^{\lambda}\right)$, where $f_{i} \in \mathcal{F}_{\lambda}$ for all $i \in[T]$.

3. $\mathrm{sk}_{f_{i}} \leftarrow \mathrm{KG}\left(\mathrm{msk}, f_{i}\right)$ for all $i \in[T]$.

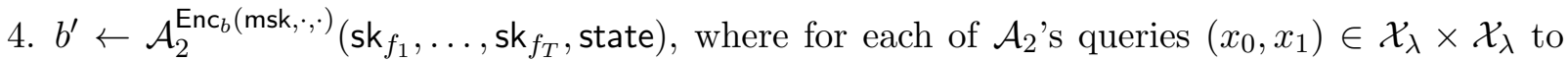
$\mathrm{Enc}_{b}(\mathrm{msk}, \cdot, \cdot)$ it holds that $f_{i}\left(x_{0}\right)=f_{i}\left(x_{1}\right)$ for all $i \in[T]$.

5. Output $b^{\prime}$.

Such a scheme is selective-function message private if it is $T$-selective-function message private for all polynomials $T=T(\lambda)$.

Known constructions. Private-key functional encryption schemes that satisfy the notions presented in Definitions 2.4 and 2.5 (and support circuits of any a-priori bounded polynomial size) are known to exist based on various assumptions. The known schemes are in fact public-key schemes, which are in particular private-key ones.

Specifically, a public-key scheme that satisfies the notion of 1-selective-function message privacy was constructed by Gorbunov, Vaikuntanathan and Wee [GVW12] under the sole assumption that public-key encryption exists. In the private-key setting, their transformation can in fact rely on any private-key encryption scheme (and thus on any one-way function). By assuming, in addition, the existence of a pseudorandom generator computable by small-depth circuits (which is known to be implied by most concrete intractability assumptions), they construct a scheme that satisfies the notion of $T$-selective-function message privacy for any predetermined polynomial $T=T(\lambda)$. However, the length of the ciphertexts in their scheme grows linearly with $T$ and with an upper bound on the circuit size of the functions that the scheme allows (which also has to be known ahead of time). Goldwasser et al. $\left[\mathrm{GKP}^{+} 13\right]$ showed that based on the Learning with Errors (LWE) assumption, $T$-selective-function message privacy can be achieved where the ciphertext size grows with $T$ and with a bound on the depth of allowed functions.

In addition, schemes that satisfy the notion of (full) message privacy (Definition 2.4) were constructed by Boyle et al. [BCP14] and by Ananth et al. $\left[\mathrm{ABG}^{+} 13\right]$ based on differing-input obfuscation, by Waters [Wat14] based on indistinguishability obfuscation, and by Garg et al. [GGH $\left.{ }^{+} 14\right]$ based on multilinear maps. We conclude that there is a variety of constructions offering various flavors of security under various assumptions that can be used as a building block in our construction.

\subsubsection{Function Privacy}

A private-key functional-encryption scheme is function private [SSW09, $\left.\mathrm{AAB}^{+} 13, \mathrm{BS} 14\right]$ if a functional key $\mathrm{sk}_{f}$ for a function $f$ reveals no "unnecessary" information on $f$. More generally, we ask that encryptions of messages $m_{1}, \ldots, m_{T}$ together with functional keys corresponding to functions $f_{1}, \ldots, f_{T}$ reveal essentially no information other than the values $\left\{f_{i}\left(m_{j}\right)\right\}_{i, j \in[T]}$. We consider two variants of function privacy: ( $f u l l$ ) function privacy in which adversaries are fully adaptive, and selective-function function privacy in which adversaries must issue their key-generation queries in advance.

Definition 2.6 (Function privacy). A functional encryption scheme FE = (Setup, KG, Enc, Dec) over a message space $\mathcal{X}=\left\{\mathcal{X}_{\lambda}\right\}_{\lambda \in \mathbb{N}}$ and a function space $\mathcal{F}=\left\{\mathcal{F}_{\lambda}\right\}_{\lambda \in \mathbb{N}}$ is function private if for any 
probabilistic polynomial-time adversary $\mathcal{A}$ there exists a negligible function neg $(\cdot)$ such that

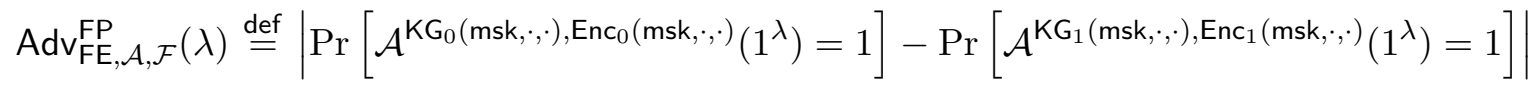

$$
\begin{aligned}
& \leq \operatorname{neg}(\lambda)
\end{aligned}
$$

for all sufficiently large $\lambda \in \mathbb{N}$, where for every $\left(f_{0}, f_{1}\right) \in \mathcal{F}_{\lambda} \times \mathcal{F}_{\lambda}$ and $\left(x_{0}, x_{1}\right) \in \mathcal{X}_{\lambda} \times \mathcal{X}_{\lambda}$ with which $\mathcal{A}$ queries the oracles KG and $\mathrm{Enc}_{b}$, respectively, it holds that $f_{0}\left(x_{0}\right)=f_{1}\left(x_{1}\right)$. Moreover, the probability is taken over the choice of msk $\leftarrow \operatorname{Setup}\left(1^{\lambda}\right)$ and the internal randomness of $\mathcal{A}$.

Definition 2.7 (Selective-function function privacy). A functional encryption scheme FE = (Setup, KG, Enc, Dec) over a message space $\mathcal{X}=\left\{\mathcal{X}_{\lambda}\right\}_{\lambda \in \mathbb{N}}$ and a function space $\mathcal{F}=\left\{\mathcal{F}_{\lambda}\right\}_{\lambda \in \mathbb{N}}$ is said T-selective-function function private, where $T=T(\lambda)$, if for any probabilistic polynomial-time adversary $\mathcal{A}=\left(\mathcal{A}_{1}, \mathcal{A}_{2}\right)$ there exists a negligible function $\operatorname{neg}(\cdot)$ such that

$$
\operatorname{Adv}_{\mathrm{FE}, \mathcal{A}, \mathcal{F}, T}^{\mathrm{sfFP}}(\lambda) \stackrel{\text { def }}{=}\left|\operatorname{Pr}\left[\operatorname{Expt}_{\mathrm{FE}, \mathcal{A}, \mathcal{F}, T}^{(0)}(\lambda)=1\right]-\operatorname{Pr}\left[\operatorname{Expt}_{\mathrm{FE}, \mathcal{A}, \mathcal{F}, T}^{(1)}(\lambda)=1\right]\right| \leq \operatorname{neg}(\lambda),
$$

for all sufficiently large $\lambda \in \mathbb{N}$, where for each $b \in\{0,1\}$ and $\lambda \in \mathbb{N}$ the random variable $\operatorname{Expt}_{\mathrm{FE}, \mathcal{A}, \mathcal{F}, T}^{(b)}(\lambda)$ is defined as follows:

1. $\mathrm{msk} \leftarrow \operatorname{Setup}\left(1^{\lambda}\right)$.

2. $\left(\left(f_{0,1}, \ldots, f_{0, T}\right),\left(f_{1,1}, \ldots, f_{1, T}\right)\right.$, state $) \leftarrow \mathcal{A}_{1}\left(1^{\lambda}\right)$, where $f_{\sigma, i} \in \mathcal{F}_{\lambda}$ for all $\sigma \in\{0,1\}$ and $i \in[T]$.

3. $\mathrm{sk}_{i}^{*} \leftarrow \mathrm{KG}\left(\mathrm{msk}, f_{b, i}\right)$ for all $i \in[T]$.

4. $b^{\prime} \leftarrow \mathcal{A}_{2}^{\text {Enc }_{b}\left(\mathrm{msk}_{, \cdot, \cdot}\right)}\left(\mathrm{sk}_{1}^{*}, \ldots, \mathrm{sk}_{T}^{*}\right.$, state $)$, where for each query $\left(x_{0}, x_{1}\right) \in \mathcal{X}_{\lambda} \times \mathcal{X}_{\lambda}$ to Enc $\mathrm{Ensk}_{b}\left(\mathrm{msk}^{\prime}, \cdot\right)$ it holds that $f_{0, i}\left(x_{0}\right)=f_{1, i}\left(x_{1}\right)$ for all $i \in[T]$.

5. Output $b^{\prime}$.

Such a scheme is selective-function function private if it is $T$-selective-function function private for all polynomials $T=T(\lambda)$.

Known constructions. Brakerski and Segev [BS14] showed how to transform any (selectivefunction or fully secure) message-private function encryption scheme into a (selective-function or fully secure, respectively) functional encryption scheme which is also function private. Thus, any instantiation of a message-private (or selective-function message private) function encryption scheme as discussed in Section 2.2.1 can be used as a building block in our construction.

\section{Private-Key Functional Encryption for Randomized Functionalities}

In this section we present a framework for capturing the security of private-key functional encryption for randomized functionalities. Our framework is inspired by that of Goyal et al. [GJK $\left.{ }^{+} 13\right]$ in the public-key setting, but takes a slightly different approach as we discuss below.

Throughout this section, we let $\mathcal{F}=\left\{\mathcal{F}_{\lambda}\right\}_{\lambda \in \mathbb{N}}$ be a family of randomized functionalities, where for every $\lambda \in \mathbb{N}$ the set $\mathcal{F}_{\lambda}$ consists of functions of the form $f: \mathcal{X}_{\lambda} \times \mathcal{R}_{\lambda} \rightarrow \mathcal{Y}_{\lambda}$. That is, such a function $f$ maps $\mathcal{X}_{\lambda}$ into $\mathcal{Y}_{\lambda}$ using randomness from $\mathcal{R}_{\lambda}$.

A private-key functional encryption scheme for a family $\mathcal{F}$ of randomized functions consists of four probabilistic polynomial-time algorithms (Setup, KG, Enc, Dec) with the same syntax that is described in Section 2.2 for deterministic functions. Although the syntax in this setting is the same as in the deterministic setting, the correctness and security requirements are more subtle. 


\subsection{Correctness and Independence}

In terms of correctness we rely on the definition of Goyal et al. [GJK ${ }^{+} 13$ ] (when adapted to the private-key setting). As discussed in Section 1.3, we ask that for any sequence of messages $x_{1}, \ldots, x_{T}$ and for any sequence of functions $f_{1}, \ldots, f_{T} \in \mathcal{F}$, it holds that the distribution obtained by encrypting $x_{1}, \ldots, x_{T}$ and then decrypting the resulting ciphertexts with functional keys corresponding to $f_{1}, \ldots, f_{T}$ is computationally indistinguishable from the distribution $\left\{f_{j}\left(x_{i} ; r_{i, j}\right)\right\}_{i, j \in[T]}$ where the $r_{i, j}$ 's are sampled independently and uniformly at random.

Definition 3.1 (Correctness). A functional encryption scheme $\Pi=$ (Setup, KG, Enc, Dec) for a family $\mathcal{F}$ of randomized functions is correct if for all sufficiently large $\lambda \in \mathbb{N}$, for every polynomial $T=T(\lambda)$, and for every $x_{1}, \ldots, x_{T} \in \mathcal{X}_{\lambda}$ and $f_{1}, \ldots, f_{T} \in \mathcal{F}_{\lambda}$, the following two distributions are computationally indistinguishable:

- $\operatorname{Real}(\boldsymbol{\lambda}) \stackrel{\text { def }}{=}\left\{\operatorname{Dec}\left(\mathrm{sk}_{f_{j}}, \mathrm{ct}_{i}\right)\right\}_{i, j \in[T]}$, where:

$-\operatorname{msk} \leftarrow \operatorname{Setup}\left(1^{\lambda}\right)$,

$-\mathrm{ct}_{i} \leftarrow \operatorname{Enc}\left(\mathrm{msk}, x_{i}\right)$ for all $i \in[T]$,

$-\mathrm{sk}_{f_{j}} \leftarrow \mathrm{KG}\left(\mathrm{msk}, f_{j}\right)$ for all $j \in[T]$.

- Ideal $(\boldsymbol{\lambda}) \stackrel{\text { def }}{=}\left\{f_{j}\left(x_{i}\right)\right\}_{i, j \in[T]}$.

As noted by Goyal et al. [GJK $\left.{ }^{+} 13\right]$, unlike in the case of deterministic functions where is suffices to define correctness for a single ciphertext and a single key, here it is essential to define correctness for multiple (possibly correlated) ciphertexts and keys. We refer the reader to $\left[\mathrm{GJK}^{+} 13\right]$ for more details.

\section{2 "Best-Possible" Message Privacy}

We consider indistinguishability-based notions for capturing message privacy in private-key functional encryption for randomized functionalities. As in the (standard) case of deterministic functions (see Section 2.2), we consider adversaries whose goal is to distinguish between encryptions of two challenge messages $x_{0}^{*}$ and $x_{1}^{*}$, when given access to an encryption oracle (as required in private-key encryption) and to functional keys of various functions. Recall that in the case of deterministic functions, the adversary is allowed to ask for functional keys for any function $f$ such that $f\left(x_{0}^{*}\right)=f\left(x_{1}^{*}\right)$.

As discussed in Section 1.3, our notions of message privacy ask that the functional encryption scheme under consideration will not add any non-negligible advantage to the (possibly non-negligible) advantage that adversaries holding a functional key for a function $f$ may already have in distinguishing between the distributions $f\left(x_{0}^{*}\right)$ and $f\left(x_{1}^{*}\right)$ to begin with. That is, given that adversaries are able to obtain a sample from the distribution $f\left(x_{0}^{*}\right)$ or from the distribution $f\left(x_{1}^{*}\right)$ using the functional key $\mathrm{sk}_{f}$, and may already have some advantage in distinguishing these distributions, we ask for "best-possible" message privacy in the sense that essentially no additional advantage can be gained.

In what follows we put forward two flavors of "best-possible" message privacy, depending on the flavor of indistinguishability guarantee that is satisfied by the function family under consideration.

Message privacy for non-adaptively-admissible functionalities. Our first notion is that of non-adaptively-admissible function families. These are families $\mathcal{F}$ such that for a randomly sampled $f \leftarrow \mathcal{F}$, no efficient adversary on input $f$ can output $x_{0}$ and $x_{1}$ and distinguish the distributions $f\left(x_{0}\right)$ and $f\left(x_{1}\right)$ with probability larger than $\Delta$ (note again that $\Delta$ does not have to be negligible). 
One possible example for such a function family is a function that on input $x$ samples a public-key $p k$ for a public-key encryption scheme, and outputs $p k$ together with a randomized encryption of $x$.

For such function families we consider a corresponding notion of message privacy in which the adversary obtains functional keys only for functions that are sampled uniformly and independently from $\mathcal{F}$. This is formally captured by the following two definitions.

Definition 3.2 (Non-adaptively-admissible function family). A family $\mathcal{F}=\left\{\mathcal{F}_{\lambda}\right\}_{\lambda \in \mathbb{N}}$ of efficientlycomputable randomized functions is $\Delta(\lambda)$-non-adaptively admissible if for any probabilistic polynomial-time algorithm $\mathcal{A}=\left(\mathcal{A}_{1}, \mathcal{A}_{2}\right)$ it holds that

$$
\operatorname{Adv}_{\mathcal{F}, \mathcal{A}}^{\text {naADM }}(\lambda) \stackrel{\text { def }}{=}\left|\operatorname{Pr}\left[\operatorname{Expt}_{\mathcal{F}, \mathcal{A}}^{\text {naADM }}(\lambda)=1\right]-\frac{1}{2}\right| \leq \Delta(\lambda)
$$

for all sufficiently large $\lambda \in \mathbb{N}$, where the random variable $\operatorname{Expt}_{\mathcal{F}, \mathcal{A}}^{\text {naADM }}(\lambda)$ is defined via the following experiment:

1. $b \leftarrow\{0,1\}, f \leftarrow \mathcal{F}_{\lambda}$.

2. $\left(x_{0}, x_{1}\right.$, state $) \leftarrow \mathcal{A}_{1}\left(1^{\lambda}, f\right)$.

3. $y=f\left(x_{b} ; r\right)$ for $r \leftarrow\{0,1\}^{*}$.

4. $b^{\prime} \leftarrow \mathcal{A}_{2}$ (y, state) .

5. If $b^{\prime}=b$ then output 1 , and otherwise output 0 .

Definition 3.3 (Message privacy; non-adaptive case). Let $\mathcal{F}=\left\{\mathcal{F}_{\lambda}\right\}_{\lambda \in \mathbb{N}}$ be a $\Delta(\lambda)$-non-adaptively admissible function family. A private-key functional encryption scheme $\Pi=$ (Setup, KG, Enc, Dec) is message private with respect to $\mathcal{F}$ if for any probabilistic polynomial-time adversary $\mathcal{A}=\left(\mathcal{A}_{1}, \mathcal{A}_{2}\right)$ and for any polynomial $T=T(\lambda)$ there exists a negligible function neg $(\lambda)$ such that

$$
\operatorname{Adv}_{\Pi, \mathcal{F}, \mathcal{A}, T}^{\mathrm{naMPRF}}(\lambda) \stackrel{\text { def }}{=}\left|\operatorname{Pr}\left[\operatorname{Expt}_{\Pi, \mathcal{F}, \mathcal{A}, T}^{\mathrm{naMPRF}}(\lambda)=1\right]-\frac{1}{2}\right| \leq T(\lambda) \cdot \Delta(\lambda)+\operatorname{neg}(\lambda),
$$

for all sufficiently large $\lambda \in \mathbb{N}$, where the random variable $\operatorname{Expt}_{\Pi, \mathcal{F}, \mathcal{A}, T}^{\mathrm{naMPF}}(\lambda)$ is defined via the following experiment:

1. $b \leftarrow\{0,1\}$, msk $\leftarrow \operatorname{Setup}\left(1^{\lambda}\right), f_{1}, \ldots, f_{T} \leftarrow \mathcal{F}_{\lambda}$.

2. sk $_{f_{i}} \leftarrow \mathrm{KG}\left(\mathrm{msk}, f_{i}\right)$ for all $i \in[T]$.

3. $\left(x_{0}^{*}, x_{1}^{*}\right.$, state $) \leftarrow \mathcal{A}_{1}^{\text {Enc }\left(\mathrm{msk}^{-}\right)}\left(1^{\lambda}, f_{1}, \ldots, f_{T}, \mathrm{sk}_{f_{1}}, \ldots, \mathrm{sk}_{f_{T}}\right)$.

4. $c^{*}=\operatorname{Enc}\left(\mathrm{msk}, x_{b}^{*}\right)$.

5. $b^{\prime} \leftarrow \mathcal{A}_{2}^{\text {Enc(msk, })}\left(c^{*}\right.$, state $)$.

6. If $b^{\prime}=b$ then output 1 , and otherwise output 0 .

Message privacy for adaptively-admissible functionalities. Our second notion is that of adaptively-admissible function families. These are families $\mathcal{F}$ such that no efficient adversary can output $f \in \mathcal{F}$ together with two inputs $x_{0}$ and $x_{1}$, and distinguish the distributions $f\left(x_{0}\right)$ and $f\left(x_{1}\right)$ with probability larger than $\Delta$. One possible example for such a function family is that of differentially private mechanisms, as discussed by Goyal et al. [GJK ${ }^{+} 13$ ]. Specifically, these are randomized functions that on any two inputs that differ on only a few of their entries, produce output distributions whose statistical distance is polynomially small (i.e., $\Delta$ is polynomial in $1 / \lambda)^{6}$.

\footnotetext{
${ }^{6}$ The definitions of differential privacy are in fact stronger than requiring small statistical distance.
} 
It is easy to observe that there are function families that are non-adaptively admissible but are not adaptively admissible. One possible example is functions of the form $f_{\mathrm{pk}}$ that are indexed by a public encryption key pk, and on input $x$ output a randomized encryption of $x$ under pk. Giving adversaries the possibility of adaptively choosing such functions, they can choose a function $f_{\mathrm{pk}}$ for which they know the corresponding decryption key sk. In this case, although for a randomly chosen pk the distributions $f_{\mathrm{pk}}\left(x_{0}\right)$ and $f_{\mathrm{pk}}\left(x_{1}\right)$ are computationally indistinguishable, they may be easily distinguishable given the randomness used by the adversary (from which it may be easy to compute the corresponding decryption key sk).

For adaptively-admissible function families we consider a corresponding notion of message privacy in which the adversary obtains functional keys for functions that are adaptively chosen from $\mathcal{F}$. This is formally captured by the following two definitions.

Definition 3.4 (Adaptively-admissible function family). A family $\mathcal{F}=\left\{\mathcal{F}_{\lambda}\right\}_{\lambda \in \mathbb{N}}$ of efficientlycomputable randomized functions is $\Delta(\lambda)$-adaptively admissible if for any probabilistic polynomialtime algorithm $\mathcal{A}=\left(\mathcal{A}_{1}, \mathcal{A}_{2}\right)$ it holds that

$$
\operatorname{Adv}_{\mathcal{F}, \mathcal{A}}^{\mathrm{aADM}}(\lambda) \stackrel{\text { def }}{=}\left|\operatorname{Pr}\left[\operatorname{Expt}_{\mathcal{F}, \mathcal{A}}^{\mathrm{aADM}}(\lambda)=1\right]-\frac{1}{2}\right| \leq \Delta(\lambda)
$$

for all sufficiently large $\lambda \in \mathbb{N}$, where the random variable $\operatorname{Expt}_{\mathcal{F}, \mathcal{A}}^{\mathrm{aADM}}(\lambda)$ is defined via the following experiment:

1. $b \leftarrow\{0,1\}$.

2. $\left(f, x_{0}, x_{1}\right.$, state $) \leftarrow \mathcal{A}_{1}\left(1^{\lambda}\right)$, where $f \in \mathcal{F}_{\lambda}$.

3. $y=f\left(x_{b} ; r\right)$ for $r \leftarrow\{0,1\}^{*}$.

4. $b^{\prime} \leftarrow \mathcal{A}_{2}$ (y, state) .

5. If $b^{\prime}=b$ then output 1 , and otherwise output 0 .

Definition 3.5 (Message privacy; adaptively-admissible case). Let $\mathcal{F}=\left\{\mathcal{F}_{\lambda}\right\}_{\lambda \in \mathbb{N}}$ be a $\Delta(\lambda)$ adaptively admissible function family. A private-key functional encryption scheme $\Pi=$ (Setup, $\mathrm{KG}$, Enc, Dec) is message private with respect to $\mathcal{F}$ if for any probabilistic polynomial-time adversary $\mathcal{A}=\left(\mathcal{A}_{1}, \mathcal{A}_{2}\right)$ that issues at most $T=T(\lambda)$ key-generation queries there exists a negligible function $\operatorname{neg}(\lambda)$ such that

$$
\operatorname{Adv}_{\Pi, \mathcal{F}, \mathcal{A}}^{\mathrm{aMPRF}}(\lambda) \stackrel{\text { def }}{=}\left|\operatorname{Pr}\left[\operatorname{Expt}_{\Pi, \mathcal{F}, \mathcal{A}}^{\mathrm{aMPRF}}(\lambda)=1\right]-\frac{1}{2}\right| \leq T(\lambda) \cdot \Delta(\lambda)+\operatorname{neg}(\lambda),
$$

for all sufficiently large $\lambda \in \mathbb{N}$, where the random variable $\operatorname{Expt}_{\Pi, \mathcal{F}, \mathcal{A}}^{\mathrm{aMPRF}}(\lambda)$ is defined via the following experiment:

1. $b \leftarrow\{0,1\}, \operatorname{msk} \leftarrow \operatorname{Setup}\left(1^{\lambda}\right)$.

2. $\left(x_{0}^{*}, x_{1}^{*}\right.$, state $) \leftarrow \mathcal{A}_{1}^{\mathrm{Enc}(\mathrm{msk}, \cdot), \mathrm{KG}(\mathrm{msk}, \cdot)}\left(1^{\lambda}\right)$.

3. $c^{*}=\operatorname{Enc}\left(\mathrm{msk}, x_{b}^{*}\right)$.

4. $b^{\prime} \leftarrow \mathcal{A}_{2}^{\text {Enc(msk, }), \mathrm{KG}(\mathrm{msk}, \cdot)}\left(c^{*}\right.$, state $)$.

5. If $b^{\prime}=b$ then output 1 , and otherwise output 0 .

\section{Our Functional Encryption Scheme}

In this section we present our construction of a private-key functional encryption scheme for randomized functionalities. Let $\mathcal{F}=\left\{\mathcal{F}_{\lambda}\right\}_{\lambda \in \mathbb{N}}$ be a family of randomized functionalities, where for 
every $\lambda \in \mathbb{N}$ the set $\mathcal{F}_{\lambda}$ consists of functions of the form $f: \mathcal{X}_{\lambda} \times \mathcal{R}_{\lambda} \rightarrow \mathcal{Y}_{\lambda}$ (i.e., $f$ maps $\mathcal{X}_{\lambda}$ into $\mathcal{Y}_{\lambda}$ using randomness from $\mathcal{R}_{\lambda}$ ). Our construction relies on the following building blocks:

1. A private-key functional encryption scheme FE = (FE.Setup, FE.KG, FE.Enc, FE.Dec).

2. A pseudorandom function family PRF $=$ (PRF.Gen, PRF.Eval). We assume that for every $\lambda \in \mathbb{N}$ and for every key $K$ that is produced by PRF.Gen $\left(1^{\lambda}\right)$, it holds that PRF.Eval $(K, \cdot):\{0,1\}^{\lambda} \rightarrow$ $\mathcal{R}_{\lambda}$.

As discussed in Section 1.1, we assume that the scheme FE is sufficiently expressive in the sense that it supports the function family $\mathcal{F}$ (when viewed as a family of single-input deterministic functions), the evaluation procedure of the pseudorandom function family PRF, and a few additional basic operations (such as conditional statements). Our scheme $\Pi=$ (Setup, KG, Enc, Dec) is defined as follows.

- The setup algorithm. On input the security parameter $1^{\lambda}$ the setup algorithm Setup samples FE.msk $\leftarrow$ FE.Setup $\left(1^{\lambda}\right)$, and outputs msk $=$ FE.msk.

- The key-generation algorithm. On input the master secret key msk and a function $f \in \mathcal{F}_{\lambda}$, the key-generation algorithm KG samples $K \leftarrow$ PRF.Gen $\left(1^{\lambda}\right)$ and outputs $\mathrm{sk}_{f} \leftarrow$ FE.KG(msk, Left $f, K)$, where Left $_{f, K}$ is a deterministic function that is defined in Figure 1.

- The encryption algorithm. On input the master secret key msk and a message $x \in \mathcal{X}_{\lambda}$, the encryption algorithm Enc samples $s \leftarrow\{0,1\}^{\lambda}$ and outputs ct $\leftarrow$ FE.Enc $(\operatorname{msk},(x, \perp, s, \perp)$ ).

- The decryption algorithm. On input a functional key $\mathrm{sk}_{f}$ and a ciphertext $\mathrm{ct}$, the decryption algorithm Dec outputs FE.Dec $\left(\mathrm{sk}_{f}, \mathrm{ct}\right)$.

$$
\begin{aligned}
& \text { Left }_{\boldsymbol{f}, \boldsymbol{K}}\left(\boldsymbol{x}_{\boldsymbol{L}}, \boldsymbol{x}_{\boldsymbol{R}}, \boldsymbol{s}, \boldsymbol{z}\right) \text { : } \\
& \text { 1. Let } r=\text { PRF.Eval }(K, s) . \\
& \text { 2. Output } f\left(x_{L} ; r\right) .
\end{aligned}
$$
Right $_{f, K}\left(x_{L}, x_{R}, s, z\right)$ :
1. Let $r=\operatorname{PRF} . \operatorname{Eval}(K, s)$.
2. Output $f\left(x_{R} ; r\right)$.

Figure 1: The functions Left $f_{f, K}$ and Right $_{f, K}$. The function Left ${ }_{f, K}$ is used by the actual scheme, whereas the function Right ${ }_{f, K}$ is used in the proofs of its security.

The correctness and independence of the above scheme with respect to any family of randomized functionalities follows in a straightforward manner from the correctness of the underlying functional encryption scheme FE and the assumption that PRF is a pseudorandom function family (in fact, it suffices that PRF is a weak pseudorandom function family). Specifically, consider a sequence of messages $x_{1}, \ldots, x_{T}$ and a sequence of functions $f_{1}, \ldots, f_{T}$. As the encryption FE.Enc(msk, $\left.\left(x_{i}, \perp, s_{i}, \perp\right)\right)$ of each message $x_{i}$ uses a uniformly sampled $s_{i} \in\{0,1\}^{\lambda}$, and the functional key for a function $f_{j}$ contains a freshly sampled key $K_{j}$ for the pseudorandom function family, the distribution $\left\{f_{j}\left(x_{i} ;\right.\right.$ PRF.Eval $\left.\left(K_{j}, s_{i}\right)\right\}$ is computationally indistinguishable from the distribution $\left\{f_{j}\left(x_{i} ; r_{i, j}\right)\right\}$, where the $r_{i, j}$ 's are sampled independently and uniformly at random.

The following two theorems capture the security of the scheme. These theorems (which are proved in Sections 4.1 and 4.2) state that under suitable assumptions on the underlying building blocks, the scheme is message private for non-adaptively-admissible randomized functionalities and for adaptively-admissible randomized functionalities.

Theorem 4.1. Assuming that PRF is a pseudorandom function family and that FE is selectivefunction function private, then $\Pi$ is message private for non-adaptively-admissible randomized functionalities. 
Theorem 4.2. Assuming that PRF is a puncturable pseudorandom function family and that FE is function private, then $\Pi$ is message private for adaptively-admissible randomized functionalities.

As discussed in Sections 2.1 and 2.2, Theorems 4.1 and 4.2 can be instantiated based on a variety of known pseudorandom function families and functional encryption schemes. In particular, Theorem 4.1 can be based on the minimal assumption that a selective-function message-private functional encryption scheme exists, and Theorem 4.2 can be based on the minimal assumption that a message-private functional encryption scheme exists.

\subsection{Proof of Theorem 4.1}

We prove that the scheme $\Pi$ is message private for non-adaptively-admissible functionalities (see Definition 3.2) based on the assumptions that PRF is a pseudorandom function family and that FE is selective-function function private (see Definition 2.7).

Let $\mathcal{A}$ be a probabilistic polynomial-time adversary that receives functional keys for at most $T=T(\lambda)$ functions (note that $T$ may be any polynomial and is not fixed in advance), and let $\mathcal{F}$ be a $\Delta$-non-adaptively admissible family of randomized functionalities. We denote by $f_{1}, \ldots, f_{T}$ the functions for which $\mathcal{A}$ receives functional keys.

We present a sequence of experiments and upper bound $\mathcal{A}$ 's advantage in distinguishing each two consecutive experiments. Each two consecutive experiments differ either in the description of their encryption oracle or in the description of their key-generation oracle. The first experiment is the experiment $\operatorname{Expt}_{\Pi, \mathcal{F}, \mathcal{A}, T}^{\mathrm{naMPRF}}(\lambda)$ (see Definition 3.3), and the last experiment is completely independent of the bit $b$. This enables us to prove that there exists a negligible function neg $(\cdot)$ such that

$$
\operatorname{Adv}_{\Pi, \mathcal{F}, \mathcal{A}, T}^{\mathrm{naMPRF}}(\lambda) \stackrel{\text { def }}{=}\left|\operatorname{Pr}\left[\operatorname{Expt}_{\Pi, \mathcal{F}, \mathcal{A}, T}^{\mathrm{naMPRF}}(\lambda)=1\right]-\frac{1}{2}\right| \leq T(\lambda) \cdot \Delta(\lambda)+\operatorname{neg}(\lambda)
$$

for all sufficiently large $\lambda \in \mathbb{N}$. Throughout the proof we use, in addition to Left $_{f, K}$ and Right $f, K$ that are defined in Figure 1, the algorithm OutputZ that is described in Figure 2. In what follows

\section{OutputZ( $\left.x_{L}, x_{R}, s, z\right)$}

1. Output $z$.

Figure 2: The function OutputZ.

we describe the experiments, and we refer the reader to Table 1 for a high-level overview of the differences between them.

Experiment $\mathcal{H}^{(0)}(\boldsymbol{\lambda})$. This is the experiment $\operatorname{Expt}_{\Pi, \mathcal{F}, \mathcal{A}, T}^{\mathrm{naMPRF}}(\lambda)$ (see Definition 3.3).

Experiment $\mathcal{H}^{(1)}(\lambda)$. This experiment is obtained from the experiment $\mathcal{H}^{(0)}(\lambda)$ by modifying the encryption oracle and the distribution of the challenge ciphertext as follows. The encryption oracle on input $x$ samples $s \leftarrow\{0,1\}^{\lambda}$ and outputs ct $\leftarrow$ FE.Enc(msk, $\left.(x, x, s, \perp)\right)$ instead of ct $\leftarrow$ FE.Enc $(m s k,(x, \perp, s, \perp))$. Similarly, the challenge ciphertext is computed by sampling $s^{*} \leftarrow\{0,1\}^{\lambda}$ and outputting ct* $\leftarrow$ FE.Enc $\left(\right.$ msk, $\left.\left(x_{b}^{*}, x_{1}^{*}, s^{*}, \perp\right)\right)$ instead of ct* $\leftarrow$ FE.Enc $\left(\right.$ msk, $\left.\left(x_{b}^{*}, \perp, s^{*}, \perp\right)\right)$.

Note that for each function $f \in\left\{f_{1}, \ldots, f_{T}\right\}$ with an associated PRF key $K$, for the deterministic function Left $f_{, K}$ it holds that $\operatorname{Left}_{f, K}(x, x, s, \perp)=\operatorname{Left}_{f, K}(x, \perp, s, \perp$ ) (and similarly for the challenge ciphertext). Therefore, the selective-function message privacy of the underlying scheme FE (with respect to deterministic functions) guarantees that the adversary $\mathcal{A}$ has only a negligible advantage in distinguishing experiments $\mathcal{H}^{(0)}$ and $\mathcal{H}^{(1)}$. Specifically, let $\mathcal{F}^{\prime}$ denote the family of deterministic 


\begin{tabular}{|c|c|c|c|}
\hline Experiment & Encryption oracle & Challenge ciphertext & Functional keys \\
\hline $\mathcal{H}^{(0)}$ & $(x, \perp, s, \perp)$ & $\left(x_{b}^{*}, \perp, s^{*}, \perp\right)$ & Left $_{f, K}$ \\
\hline $\mathcal{H}^{(1)}$ & $(x, x, s, \perp)$ & $\left(x_{b}^{*}, x_{1}^{*}, s^{*}, \perp\right)$ & Left $_{f, K}$ \\
\hline \multirow{2}{*}{$\mathcal{H}^{(2, i)}$} & \multirow{2}{*}{$(x, x, s, \perp)$} & \multirow{2}{*}{$\left(x_{b}^{*}, x_{1}^{*}, s^{*}, \perp\right)$} & Keys $1, \ldots, i-1:$ Right $_{f, K}$ \\
\hline & & & Keys $i, \ldots, T:$ Left $_{f, K}$ \\
\hline \multirow[t]{2}{*}{$\mathcal{H}^{(3, i)}$} & $\left(x, x, s, f_{i}\left(x ; \operatorname{PRF}_{K_{i}}(s)\right)\right)$ & $\left(x_{b}^{*}, x_{1}^{*}, s^{*}, f_{i}\left(x_{b}^{*} ; \operatorname{PRF}_{K_{i}}\left(s^{*}\right)\right)\right)$ & Keys $_{1}, \ldots, i-1$ : Right $_{f, K}$ \\
\hline & & & Keys $i, \ldots, T:$ Left $_{f, K}$ \\
\hline \multirow{3}{*}{$\mathcal{H}^{(4, i)}$} & \multirow{3}{*}{$\left(x, x, s, f_{i}\left(x ; \operatorname{PRF}_{K_{i}}(s)\right)\right)$} & \multirow{3}{*}{$\left(x_{b}^{*}, x_{1}^{*}, s^{*}, f_{i}\left(x_{b}^{*} ; \operatorname{PRF}_{K_{i}}\left(s^{*}\right)\right)\right)$} & $\operatorname{Keys}_{1}, \ldots, i-1$ : $\operatorname{Right}_{f, K}$ \\
\hline & & & Key $i$ : OutputZ \\
\hline & & & Keys $i+1, \ldots, T$ : Left $f, K$ \\
\hline \multirow[t]{2}{*}{$\mathcal{H}^{(5, i)}$} & \multirow[t]{2}{*}{$\left(x, x, s, f_{i}(x ; r)\right)$} & \multirow[t]{2}{*}{$\left(x_{b}^{*}, x_{1}^{*}, s^{*}, f_{i}\left(x_{b}^{*} ; r^{*}\right)\right)$} & $\begin{array}{l}\text { Keys } 1, \ldots, i-1: \text { Right }_{f, K} \\
\text { Key } i \text { : OutputZ }\end{array}$ \\
\hline & & & Keys $i+1, \ldots, T$ : Left $_{f, K}$ \\
\hline \multirow{3}{*}{$\mathcal{H}^{(6, i)}$} & \multirow{3}{*}{$\left(x, x, s, f_{i}(x ; r)\right)$} & \multirow{3}{*}{$\left(x_{b}^{*}, x_{1}^{*}, s^{*}, f_{i}\left(x_{1}^{*} ; r^{*}\right)\right)$} & $\operatorname{Keys}_{1} 1, \ldots, i-1$ : $\operatorname{Right}_{f, K}$ \\
\hline & & & Key $i$ : OutputZ \\
\hline & & & Keys $i+1, \ldots, T$ : Left $f, K$ \\
\hline \multirow{3}{*}{$\mathcal{H}^{(7, i)}$} & \multirow{3}{*}{$\left(x, x, s, f_{i}\left(x ; \operatorname{PRF}_{K_{i}}(s)\right)\right)$} & \multirow{3}{*}{$\left(x_{b}^{*}, x_{1}^{*}, s^{*}, f_{i}\left(x_{1}^{*} ; \operatorname{PRF}_{K_{i}}\left(s^{*}\right)\right)\right)$} & Keys $1, \ldots, i-1$ : Right $_{f, K}$ \\
\hline & & & Key $i$ : OutputZ \\
\hline & & & Keys $i+1, \ldots, T:$ Left $_{f, K}$ \\
\hline \multirow{2}{*}{$\mathcal{H}^{(8, i)}$} & \multirow{2}{*}{$\left(x, x, s, f_{i}\left(x ; \operatorname{PRF}_{K_{i}}(s)\right)\right)$} & \multirow{2}{*}{$\left(x_{b}^{*}, x_{1}^{*}, s^{*}, f_{i}\left(x_{1}^{*} ; \operatorname{PRF}_{K_{i}}\left(s^{*}\right)\right)\right)$} & Keys $1, \ldots, i$ : Right $_{f, K}$ \\
\hline & & & Keys $i+1, \ldots, T:$ Left $_{f, K}$ \\
\hline $\mathcal{H}^{(9)}$ & $(x, x, s, \perp)$ & $\left(x_{1}^{*}, x_{1}^{*}, s^{*}, \perp\right)$ & Right $_{f, K}$ \\
\hline
\end{tabular}

Table 1: The differences between the experiments $\mathcal{H}^{(0)}, \ldots, \mathcal{H}^{(9)}$. 
functions Left ${ }_{f, K}$ and Right ${ }_{f, K}$ for every $f \in \mathcal{F}$ and PRF key $K$ (as defined in Figure 1) as well as the function OutputZ (as defined in Figure 2). In Appendix B we prove the following claim:

Claim 4.3. There exists a probabilistic polynomial-time adversary $\mathcal{B}^{(0) \rightarrow(1)}$ such that

$$
\left|\operatorname{Pr}\left[\mathcal{H}^{(0)}(\lambda)=1\right]-\operatorname{Pr}\left[\mathcal{H}^{(1)}(\lambda)=1\right]\right| \leq \operatorname{Adv}_{\mathrm{FE}, \mathcal{F}^{\prime}, \mathcal{B}^{(0) \rightarrow(1)}, T}^{\mathrm{sfMP}}(\lambda) .
$$

Experiment $\mathcal{H}^{(2, i)}(\lambda)$ where $i \in[\boldsymbol{T}+1]$. This experiment is obtained from the experiment $\mathcal{H}^{(1)}(\lambda)$ by modifying the distribution of the functional keys as follows. The functional keys for $f_{1}, \ldots, f_{i-1}$ are generated as sk $\leftarrow$ FE.KG(msk, Right $\left._{f, K}\right)$ instead of as sk $f \leftarrow$ FE.KG $\left(m s k\right.$, Left $\left._{f, K}\right)$ (where Right $f_{, K}$ is defined in Figure 1), and the functional keys for $f_{i}, \ldots, f_{T}$ are generated as before

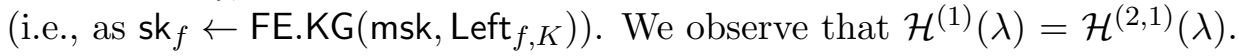

Experiment $\mathcal{H}^{(3, i)}(\boldsymbol{\lambda})$ where $\boldsymbol{i} \in[\boldsymbol{T}]$. This experiment is obtained from the experiment $\mathcal{H}^{(2, i)}(\lambda)$ by modifying the encryption oracle and the distribution of the challenge ciphertext as follows. The encryption oracle on input $x$ samples $s \leftarrow\{0,1\}^{\lambda}$ and outputs ct $\leftarrow$ FE.Enc $(m s k,(x, x, s, z))$, where $z=f_{i}\left(x ; \operatorname{PRF} . \operatorname{Eval}\left(K_{i}, s\right)\right)$, instead of ct $\leftarrow$ FE.Enc $(m s k,(x, x, s, \perp))$. Similarly, the challenge ciphertext is computed by sampling $s^{*} \leftarrow\{0,1\}^{\lambda}$ and outputting ct $\leftarrow$ FE.Enc $\left(\mathrm{msk},\left(x_{b}^{*}, x_{1}^{*}, s^{*}, z^{*}\right)\right)$, where $z^{*}=f_{i}\left(x_{b}^{*}\right.$; PRF.Eval $\left.\left(K_{i}, s^{*}\right)\right)$, instead of ct $\leftarrow$ FE.Enc $\left(\operatorname{msk},\left(x_{b}^{*}, x_{1}^{*}, s^{*}, \perp\right)\right)$.

Note that for function $f \in\left\{f_{1}, \ldots, f_{T}\right\}$ with an associated PRF key $K$, for the deterministic functions Left $f_{, K}$ and $\operatorname{Right}_{f, K}$ it holds that Left $f_{, K}(x, x, s, z)=\operatorname{Left}_{f, K}(x, x, s, \perp)$ and $\operatorname{Right}_{f, K}(x, x, s, z)$ $=\operatorname{Right}_{f, K}(x, x, s, \perp$ ) (and similarly for the challenge ciphertext). Therefore, the selective-function message privacy of the underlying scheme FE (with respect to deterministic functions) guarantees that the adversary $\mathcal{A}$ has only a negligible advantage in distinguishing experiments $\mathcal{H}^{(2, i)}$ and $\mathcal{H}^{(3, i)}$. In Appendix B we prove the following claim:

Claim 4.4. For every $i \in[T]$ there exists a probabilistic polynomial-time adversary $\mathcal{B}^{(2, i) \rightarrow(3, i)}$ such that

$$
\left|\operatorname{Pr}\left[\mathcal{H}^{(2, i)}(\lambda)=1\right]-\operatorname{Pr}\left[\mathcal{H}^{(3, i)}(\lambda)=1\right]\right| \leq \operatorname{Adv}_{\mathrm{FE}, \mathcal{F}^{\prime}, \mathcal{B}(2, i) \rightarrow(3, i), T}^{\mathrm{sfMP}}(\lambda) .
$$

Experiment $\mathcal{H}^{(4, i)}(\boldsymbol{\lambda})$ where $\boldsymbol{i} \in[\boldsymbol{T}]$. This experiment is obtained from the experiment $\mathcal{H}^{(3, i)}(\lambda)$ by modifying the distribution of the functional key for the $i$ th function $f_{i}$. Specifically, the functional key for $f_{i}$ is computed as sk $f_{i} \leftarrow$ FE.KG(msk, OutputZ) instead of sk $f_{i} \leftarrow$ FE.KG(msk, Left $\left.f_{i}, K_{i}\right)$, where the function OutputZ is defined in Figure 1.

Note that the encrypted values are all of the form $\left(x, x, s, f_{i}\left(x ; \operatorname{PRF} . \operatorname{Eval}\left(K_{i}, s\right)\right)\right)$ or of the form $\left(x_{b}^{*}, x_{1}^{*}, s^{*}, f_{i}\left(x_{b}^{*}\right.\right.$; PRF.Eval $\left.\left.\left(K_{i}, s^{*}\right)\right)\right)$, and therefore OutputZ $\left(x, x, s, f_{i}\left(x ; \operatorname{PRF} . \operatorname{Eval}\left(K_{i}, s\right)\right)\right)=$ Left $_{f_{i}, K_{i}}\left(x, x, s, f_{i}\left(x ;\right.\right.$ PRF.Eval $\left.\left.\left(K_{i}, s\right)\right)\right)$ and similarly OutputZ $\left(x_{b}^{*}, x_{1}^{*}, s^{*}, f_{i}\left(x_{b}^{*} ; \operatorname{PRF} . \operatorname{Eval}\left(K_{i}, s^{*}\right)\right)\right)=$ Left $_{f_{i}, K_{i}}\left(x_{b}^{*}, x_{1}^{*}, s^{*}, f_{i}\left(x_{b}^{*}\right.\right.$; PRF.Eval $\left.\left.\left(K_{i}, s^{*}\right)\right)\right)$. Therefore, the selective-function function privacy of the underlying scheme FE (with respect to deterministic functions) guarantees that the adversary $\mathcal{A}$ has only a negligible advantage in distinguishing experiments $\mathcal{H}^{(3, i)}$ and $\mathcal{H}^{(4, i)}$. In Appendix B we prove the following claim:

Claim 4.5. For every $i \in[T]$ there exists a probabilistic polynomial-time adversary $\mathcal{B}^{(3, i) \rightarrow(4, i)}$ such that

$$
\left|\operatorname{Pr}\left[\mathcal{H}^{(3, i)}(\lambda)=1\right]-\operatorname{Pr}\left[\mathcal{H}^{(4, i)}(\lambda)=1\right]\right| \leq \operatorname{Adv}_{\mathrm{FE}, \mathcal{F}^{\prime}, \mathcal{B}^{(3, i) \rightarrow(4, i)}, T}^{\mathrm{sfFP}}(\lambda)
$$


Experiment $\mathcal{H}^{(5, i)}(\boldsymbol{\lambda})$ where $\boldsymbol{i} \in[\boldsymbol{T}]$. This experiment is obtained from the experiment $\mathcal{H}^{(4, i)}(\lambda)$ by modifying the encryption oracle and the distribution of the challenge ciphertext as follows. The encryption oracle on input $x$ outputs ct $\leftarrow$ FE.Enc $(m s k,(x, x, s, z))$, where $z=f_{i}(x ; r)$ for a fresh and uniformly sampled value $r$ instead of $z=f_{i}\left(x ; \operatorname{PRF}\right.$.Eval $\left(K_{i}, s\right)$. Similarly, the challenge ciphertext is computed as ct* $\leftarrow$ FE.Enc $\left(\mathrm{msk},\left(x_{b}^{*}, x_{1}^{*}, s^{*}, z^{*}\right)\right)$, where $z^{*}=f_{i}\left(x_{b}^{*} ; r^{*}\right)$ for a fresh and uniformly sampled value $r^{*}$ instead of $z^{*}=f_{i}\left(x_{b}^{*} ; \operatorname{PRF}\right.$.Eval $\left.\left(K_{i}, s^{*}\right)\right)$.

The pseudorandomness of PRF.Eval $\left(K_{i}, \cdot\right)$ guarantees that the adversary $\mathcal{A}$ has only a negligible advantage in distinguishing experiments $\mathcal{H}^{(4, i)}$ and $\mathcal{H}^{(5, i)}$. In Appendix B we prove the following claim:

Claim 4.6. For every $i \in[T]$ there exists a probabilistic polynomial-time adversary $\mathcal{B}^{(4, i) \rightarrow(5, i)}$ such that

$$
\left|\operatorname{Pr}\left[\mathcal{H}^{(4, i)}(\lambda)=1\right]-\operatorname{Pr}\left[\mathcal{H}^{(5, i)}(\lambda)=1\right]\right| \leq \operatorname{Adv}_{\mathrm{PRF}, \mathcal{B}^{(4, i) \rightarrow(5, i)}}(\lambda)
$$

Experiment $\mathcal{H}^{(6, i)}(\boldsymbol{\lambda})$ where $\boldsymbol{i} \in[\boldsymbol{T}]$. This experiment is obtained from the experiment $\mathcal{H}^{(5, i)}(\lambda)$ by computing the challenge ciphertext as ct* $\leftarrow$ FE.Enc $\left(\operatorname{msk},\left(x_{b}^{*}, x_{1}^{*}, s^{*}, z^{*}\right)\right)$, where $z^{*}=f_{i}\left(x_{1}^{*} ; r^{*}\right)$ instead of $z=f_{i}\left(x_{b}^{*} ; r^{*}\right)$.

The computational admissibility of the function family $\mathcal{F}$ guarantees that the advantage of the adversary $\mathcal{A}$ in distinguishing experiments $\mathcal{H}^{(5, i)}$ and $\mathcal{H}^{(6, i)}$ is at most $\Delta(\lambda)$. In Appendix $\mathrm{B}$ we prove the following claim:

Claim 4.7. For every $i \in[T]$ there exists a probabilistic polynomial-time adversary $\mathcal{B}^{(5, i) \rightarrow(6, i)}$ such that

$$
\left|\operatorname{Pr}\left[\mathcal{H}^{(5, i)}(\lambda)=1\right]-\operatorname{Pr}\left[\mathcal{H}^{(6, i)}(\lambda)=1\right]\right| \leq \operatorname{Adv}_{\mathcal{F}, \mathcal{B}^{(5, i) \rightarrow(6, i)}}^{\text {naADM }}(\lambda) \leq \Delta(\lambda)
$$

Experiment $\mathcal{H}^{(7, i)}(\boldsymbol{\lambda})$ where $\boldsymbol{i} \in[\boldsymbol{T}]$. This experiment is obtained from the experiment $\mathcal{H}^{(6, i)}(\lambda)$ by modifying the encryption oracle and the distribution of the challenge ciphertext as follows. The encryption oracle on input $x$ outputs ct $\leftarrow \operatorname{FE}$.Enc $(m s k,(x, x, s, z))$ where $z=f_{i}\left(x ; \operatorname{PRF} . \operatorname{Eval}\left(K_{i}, s\right)\right)$ instead of $z=f_{i}(x ; r)$ for a fresh and uniformly sampled value $r$. Similarly, the challenge ciphertext is computed as ct* $\leftarrow$ FE.Enc $\left(m s k,\left(x_{b}^{*}, x_{1}^{*}, s^{*}, z^{*}\right)\right)$, where $z=f_{i}\left(x_{1}^{*} ; \operatorname{PRF}\right.$.Eval $\left.\left(K_{i}, s^{*}\right)\right)$ instead of $z=f_{i}\left(x_{1}^{*} ; r^{*}\right)$ for a fresh and uniformly sampled value $r^{*}$.

The pseudorandomness of PRF.Eval $\left(K_{i}, \cdot\right)$ guarantees that the adversary $\mathcal{A}$ has only a negligible advantage in distinguishing experiments $\mathcal{H}^{(6, i)}$ and $\mathcal{H}^{(7, i)}$. The proof of the following claim is essentially identical to the proof of Claim 4.6 (see Appendix B):

Claim 4.8. For every $i \in[T]$ there exists a probabilistic polynomial-time adversary $\mathcal{B}^{(6, i) \rightarrow(7, i)}$ such that

$$
\left|\operatorname{Pr}\left[\mathcal{H}^{(6, i)}(\lambda)=1\right]-\operatorname{Pr}\left[\mathcal{H}^{(7, i)}(\lambda)=1\right]\right| \leq \operatorname{Adv}_{\mathrm{PRF}, \mathcal{B}(6, i) \rightarrow(7, i)}(\lambda) .
$$

Experiment $\mathcal{H}^{(8, i)}(\lambda)$ where $i \in[\boldsymbol{T}]$. This experiment is obtained from the experiment $\mathcal{H}^{(7, i)}(\lambda)$ by modifying the distribution of the functional key for the $i$ th function $f_{i}$. Specifically, the functional key for $f_{i}$ is computed as sk $f_{i} \leftarrow$ FE.KG(msk, Right $\left._{f_{i}, K_{i}}\right)$ instead of sk $f_{i} \leftarrow$ FE.KG(msk, OutputZ). 
As in the proof of Claim 4.5, the selective-function function privacy of the underlying scheme FE (with respect to deterministic functions) guarantees that the adversary $\mathcal{A}$ has only a negligible advantage in distinguishing experiments $\mathcal{H}^{(7, i)}$ and $\mathcal{H}^{(8, i)}$. The proof of the following claim is essentially identical to the proof of Claim 4.5 (see Appendix B):

Claim 4.9. For every $i \in[T]$ there exists a probabilistic polynomial-time adversary $\mathcal{B}^{(7, i) \rightarrow(8, i)}$ such that

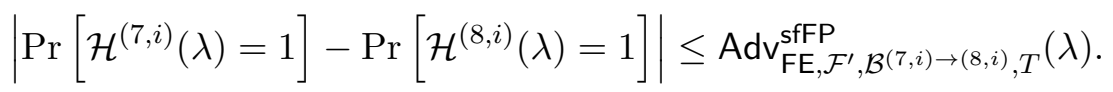

Next, we observe that the experiment $\mathcal{H}^{(2, i+1)}(\lambda)$ is obtained from the experiment $\mathcal{H}^{(8, i)}(\lambda)$ by modifying the encryption oracle and the distribution of the challenge ciphertext as follows. The encryption oracle on input $x$ outputs ct $\leftarrow$ FE.Enc $(\mathrm{msk},(x, x, s, \perp))$ instead of ct $\leftarrow \mathrm{FE}$. Enc $(\mathrm{msk}$, $(x, x, s, z))$ where $z=f_{i}\left(x ;\right.$ PRF.Eval $\left.\left(K_{i}, s\right)\right)$. Similarly, the challenge ciphertext is computed using $z^{*}=\perp$ instead of $z^{*}=f_{i}\left(x_{1}^{*}\right.$; PRF.Eval $\left.\left(K_{i}, s^{*}\right)\right)$.

Note that for each function $f \in\left\{f_{1}, \ldots, f_{T}\right\}$ with an associated PRF key $K$, for the deterministic functions $\operatorname{Left}_{f, K}$ and $\operatorname{Right}_{f, K}$ it holds that $\operatorname{Left}_{f, K}(x, x, s, \perp)=\operatorname{Left}_{f, K}(x, x, s, z)$ and $\operatorname{Right}_{f, K}(x, x, s, \perp)=\operatorname{Right}_{f, K}(x, x, s, z)$ (and similarly for the challenge ciphertext). Therefore, the selective-function message privacy of the underlying scheme FE (with respect to deterministic functions) guarantees that the adversary $\mathcal{A}$ has only a negligible advantage in distinguishing experiments $\mathcal{H}^{(8, i)}$ and $\mathcal{H}^{(2, i+1)}$. The proof of the following claim is essentially identical to the proof of Claim 4.4 (see Appendix B):

Claim 4.10. For every $i \in[T]$ there exists a probabilistic polynomial-time adversary $\mathcal{B}^{(8, i) \rightarrow(2, i+1)}$ such that

$$
\left|\operatorname{Pr}\left[\mathcal{H}^{(8, i)}(\lambda)=1\right]-\operatorname{Pr}\left[\mathcal{H}^{(2, i+1)}(\lambda)=1\right]\right| \leq \operatorname{Adv}_{\mathrm{FE}, \mathcal{F}^{\prime}, \mathcal{B}^{(8, i) \rightarrow(2, i+1)}, T}^{\mathrm{sfMP}}(\lambda) .
$$

Experiment $\mathcal{H}^{(\mathbf{9})}(\boldsymbol{\lambda})$. This experiment is obtained from the experiment $\mathcal{H}^{(2, T+1)}(\lambda)$ by computing the challenge ciphertext as $\mathrm{ct}^{*} \leftarrow \mathrm{FE}$.Enc $\left(\mathrm{msk},\left(x_{1}^{*}, x_{1}^{*}, s^{*}, \perp\right)\right)$ instead of ct $\leftarrow \mathrm{FE}$.Enc(msk, $\left.\left(x_{b}^{*}, x_{1}^{*}, s^{*}, \perp\right)\right)$. Note that this experiment is completely independent of the bit $b$, and therefore $\operatorname{Pr}\left[\mathcal{H}^{(9)}(\lambda)=1\right]=1 / 2$.

In addition, note that for every function $f \in\left\{f_{1}, \ldots, f_{T}\right\}$ with an associated PRF key $K$, for the deterministic function $\operatorname{Right}_{f, K}$ it holds that $\operatorname{Right}_{f, K}\left(x_{b}^{*}, x_{1}^{*}, s^{*}, \perp\right)=\operatorname{Right}_{f, K}\left(x_{1}^{*}, x_{1}^{*}, s^{*}, \perp\right)$. Therefore, the selective-function message privacy of the underlying scheme FE (with respect to deterministic functions) guarantees that the adversary $\mathcal{A}$ has only a negligible advantage in distinguishing experiments $\mathcal{H}^{(8, i)}$ and $\mathcal{H}^{(2, i+1)}$. The proof of the following claim is essentially identical to the proof of Claim 4.3 (see Appendix B):

Claim 4.11. There exists a probabilistic polynomial-time adversary $\mathcal{B}^{(2, T+1) \rightarrow(9)}$ such that

$$
\left|\operatorname{Pr}\left[\mathcal{H}^{(2, T+1)}(\lambda)=1\right]-\operatorname{Pr}\left[\mathcal{H}^{(9)}(\lambda)=1\right]\right| \leq \operatorname{Adv}_{\mathrm{FE}, \mathcal{F}^{\prime}, \mathcal{B}(2, T+1) \rightarrow(9), T}^{\mathrm{sfPP}}(\lambda)
$$

Finally, putting together Claims $4.3-4.11$ with the facts that $\operatorname{Expt}_{\Pi, \mathcal{F}, \mathcal{A}, T}^{\mathrm{naMPRF}}(\lambda)=\mathcal{H}^{(0)}(\lambda), \mathcal{H}^{(1)}(\lambda)=$ 
$\mathcal{H}^{(2,1)}(\lambda)$ and $\operatorname{Pr}\left[\mathcal{H}^{(9)}(\lambda)=1\right]=1 / 2$, we observe that

$$
\begin{aligned}
\operatorname{Adv}_{\Pi, \mathcal{F}, \mathcal{A}, T}^{\text {naMPR }} \stackrel{\text { def }}{=} & \operatorname{Pr}\left[\operatorname{Expt}_{\Pi, \mathcal{F}, \mathcal{A}, T}^{\text {naMPR }}(\lambda)=1\right]-\frac{1}{2} \mid \\
= & \left|\operatorname{Pr}\left[\mathcal{H}^{(0)}(\lambda)=1\right]-\operatorname{Pr}\left[\mathcal{H}^{(9)}(\lambda)=1\right]\right| \\
\leq & \left|\operatorname{Pr}\left[\mathcal{H}^{(0)}(\lambda)=1\right]-\operatorname{Pr}\left[\mathcal{H}^{(1)}(\lambda)=1\right]\right| \\
& +\sum_{i=1}^{T} \sum_{j=2}^{7}\left|\operatorname{Pr}\left[\mathcal{H}^{(j, i)}(\lambda)=1\right]-\operatorname{Pr}\left[\mathcal{H}^{(j+1, i)}(\lambda)=1\right]\right| \\
& +\sum_{i=1}^{T}\left|\operatorname{Pr}\left[\mathcal{H}^{(8, i)}(\lambda)=1\right]-\operatorname{Pr}\left[\mathcal{H}^{(2, i+1)}(\lambda)=1\right]\right| \\
& +\left|\operatorname{Pr}\left[\mathcal{H}^{(2, T+1)}(\lambda)=1\right]-\operatorname{Pr}\left[\mathcal{H}^{(9)}(\lambda)=1\right]\right| \\
\leq & T(\lambda) \cdot \Delta(\lambda)+\operatorname{neg}(\lambda) .
\end{aligned}
$$

\subsection{Proof of Theorem 4.2}

We prove that the scheme $\Pi$ is message private for adaptively-admissible functionalities (see Definition 3.5) based on the assumptions that PRF is a puncturable pseudorandom function family and that FE is function private (see Definition 2.6).

Let $\mathcal{A}$ be a probabilistic polynomial-time adversary that issues at most $T_{1}=T_{1}(\lambda)$ pre-challenge key-generation queries, at most $T_{2}=T_{2}(\lambda)$ post-challenge key-generation queries (where $T=$ $T_{1}+T_{2}$ ), and at most $T=T(\lambda)$ encryption queries (note that $T_{1}, T_{2}$ and $T$ may be any polynomials and are not fixed in advance), and let $\mathcal{F}$ be a $\Delta$-adaptively admissible family of randomized functionalities. We denote by $f_{1}, \ldots, f_{T}$ the key-generation queries that are issued by $\mathcal{A}$.

We present a sequence of experiments and upper bound $\mathcal{A}$ 's advantage in distinguishing each two consecutive experiments. Each two consecutive experiments differ either in the distribution of their challenge ciphertexts or in the distribution of the functional keys that are produced by the key-generation oracle. The first experiment is the experiment $\operatorname{Expt}_{\Pi, \mathcal{F}, \mathcal{A}, T}^{\mathrm{aMPRF}}(\lambda)$ (see Definition 3.5), and the last experiment is completely independent of the bit $b$. This enables us to prove that there exists a negligible function $\operatorname{neg}(\cdot)$ such that

$$
\operatorname{Adv}_{\Pi, \mathcal{F}, \mathcal{A}, T}^{\mathrm{aMPRF}}(\lambda) \stackrel{\text { def }}{=}\left|\operatorname{Pr}\left[\operatorname{Expt}_{\Pi, \mathcal{F}, \mathcal{A}, T}^{\mathrm{aMPRF}}(\lambda)=1\right]-\frac{1}{2}\right| \leq T(\lambda) \cdot \Delta(\lambda)+\operatorname{neg}(\lambda)
$$

for all sufficiently large $\lambda \in \mathbb{N}$. Throughout the proof we use, in addition to the functions Left $f_{f, K}$ and Right $_{f, K}$ that were defined in Figure 1, the functions PuncOutput $Y_{f, K^{\prime}, y, s^{*}}$ and PuncOutputZ $Z_{f, K^{\prime}, s^{*}}$ that are defined in Figure 3. In what follows we describe the experiments, and we refer the reader to Table 2 for a high-level overview of the differences between them. We note that in all experiments the encryption oracle is as defined by the encryption procedure of the scheme.

Experiment $\mathcal{H}^{(0)}(\lambda)$. This is the experiment $\operatorname{Expt}_{\Pi, \mathcal{F}, \mathcal{A}}^{\mathrm{aMPRF}}(\lambda)$ (see Definition 3.5).

Experiment $\mathcal{H}^{(1)}(\lambda)$. This experiment is obtained from the experiment $\mathcal{H}^{(0)}(\lambda)$ by modifying the encryption oracle so that on the challenge input $\left(x_{0}^{*}, x_{1}^{*}\right)$ it samples $s^{*} \leftarrow\{0,1\}^{\lambda}$ and outputs ct $\leftarrow$ FE.Enc $\left(\right.$ msk, $\left.\left(x_{b}^{*}, x_{1}^{*}, s^{*}, \perp\right)\right)$ instead of ct $\leftarrow$ FE.Enc $\left(\mathrm{msk},\left(x_{b}^{*}, \perp, s^{*}, \perp\right)\right)$. 


\begin{tabular}{|c|c|c|c|}
\hline Experiment & $\begin{array}{l}\text { Challenge } \\
\text { ciphertext }\end{array}$ & $\begin{array}{l}\text { Key-generation oracle } \\
\quad(\text { pre-challenge) }\end{array}$ & $\begin{array}{l}\text { Key-generation oracle } \\
\quad(\text { post-challenge })\end{array}$ \\
\hline $\mathcal{H}^{(0)}$ & $\left(x_{b}^{*}, \perp, s^{*}, \perp\right)$ & Left $_{f, K}$ & Left $_{f, K}$ \\
\hline $\mathcal{H}^{(1)}$ & $\left(x_{b}^{*}, x_{1}^{*}, s^{*}, \perp\right)$ & Left $_{f, K}$ & Left $_{f, K}$ \\
\hline $\mathcal{H}^{(2, i)}$ & $\left(x_{b}^{*}, x_{1}^{*}, s^{*}, \perp\right)$ & Left $_{f, K}$ & 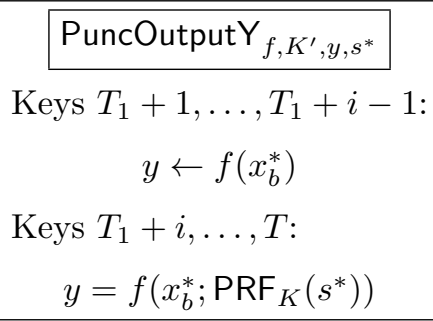 \\
\hline $\mathcal{H}^{(3, i)}$ & $\left(x_{b}^{*}, x_{1}^{*}, s^{*}, \perp\right)$ & Left $_{f, K}$ & $\begin{array}{c}\text { PuncOutputY }_{f, K^{\prime}, y, s^{*}} \\
\text { Keys } T_{1}+1, \ldots, T_{1}+i-1: \\
y \leftarrow f\left(x_{1}^{*}\right) \\
\text { Keys } T_{1}+i, \ldots, T: \\
y=f\left(x_{b}^{*} ; \operatorname{PRF}_{K}\left(s^{*}\right)\right)\end{array}$ \\
\hline $\mathcal{H}^{(4, i)}$ & $\left(x_{b}^{*}, x_{1}^{*}, s^{*}, \perp\right)$ & $\begin{array}{l}\text { Keys } 1, \ldots, i-1: \text { Right }_{f, K} \\
\text { Keys } i, \ldots, T_{1} \text { : } \text { Left }_{f, K}\end{array}$ & $\begin{array}{c}\text { PuncOutputY }_{f, K^{\prime}, y, s^{*}} \\
y \leftarrow f\left(x_{1}^{*}\right)\end{array}$ \\
\hline $\mathcal{H}^{(5, i)}$ & $\begin{array}{c}\left(x_{b}^{*}, x_{1}^{*}, s^{*}, z^{*}\right) \\
z^{*}=f_{i}\left(x_{b}^{*} ; \operatorname{PRF}_{K_{i}}\left(s^{*}\right)\right)\end{array}$ & $\begin{array}{l}\text { Keys } 1, \ldots, i-1 \text { : } \text { Right }_{f, K} \\
\text { Keys } i, \ldots, T_{1} \text { : } \text { Left }_{f, K}\end{array}$ & $\begin{array}{c}\text { PuncOutput }_{f, K^{\prime}, y, s^{*}} \\
y \leftarrow f\left(x_{1}^{*}\right)\end{array}$ \\
\hline $\mathcal{H}^{(6, i)}$ & $\begin{aligned} & \left(x_{b}^{*}, x_{1}^{*}, s^{*}, z^{*}\right) \\
z^{*}= & f_{i}\left(x_{b}^{*} ; \operatorname{PRF}_{K_{i}}\left(s^{*}\right)\right)\end{aligned}$ & $\begin{array}{l}\text { Keys } 1, \ldots, i-1 \text { : } \text { Right }_{f, K} \\
\text { Key } i \text { : PuncOutputZ } \\
\text { Keys } i+1, \ldots, T_{1}: \text { Left }_{f, K}^{\prime}, s^{*}\end{array}$ & $\begin{array}{l}\text { PuncOutputY }_{f, K^{\prime}, y, s^{*}} \\
y \leftarrow f\left(x_{1}^{*}\right)\end{array}$ \\
\hline $\mathcal{H}^{(7, i)}$ & $\begin{array}{c}\left(x_{b}^{*}, x_{1}^{*}, s^{*}, z^{*}\right) \\
z^{*}=f_{i}\left(x_{b}^{*} ; r^{*}\right)\end{array}$ & $\begin{array}{l}\text { Keys } 1, \ldots, i-1 \text { : } \text { Right }_{f, K} \\
\text { Key } i \text { : PuncOutputZ } f_{i}, K_{i}^{\prime}, s^{*} \\
\text { Keys } i+1, \ldots, T_{1} \text { : } \text { Left }_{f, K}\end{array}$ & $\begin{array}{l}\text { PuncOutputY }_{f, K^{\prime}, y, s^{*}} \\
\qquad y \leftarrow f\left(x_{1}^{*}\right)\end{array}$ \\
\hline $\mathcal{H}^{(8, i)}$ & $\begin{array}{c}\left(x_{b}^{*}, x_{1}^{*}, s^{*}, z^{*}\right) \\
z^{*}=f_{i}\left(x_{1}^{*} ; r^{*}\right)\end{array}$ & $\begin{array}{l}\text { Keys } 1, \ldots, i-1: \text { Right }_{f, K} \\
\text { Key } i \text { : PuncOutputZ } \\
f_{i}, K_{i}^{\prime}, s^{*} \\
\text { Keys } i+1, \ldots, T_{1}: \text { Left }_{f, K}\end{array}$ & $\begin{array}{l}\text { PuncOutputY }_{f, K^{\prime}, y, s^{*}} \\
\qquad y \leftarrow f\left(x_{1}^{*}\right)\end{array}$ \\
\hline $\mathcal{H}^{(9, i)}$ & $\begin{aligned} & \left(x_{b}^{*}, x_{1}^{*}, s^{*}, z^{*}\right) \\
z^{*}= & f_{i}\left(x_{1}^{*} ; \operatorname{PRF}_{K_{i}}\left(s^{*}\right)\right)\end{aligned}$ & $\begin{array}{l}\text { Keys } 1, \ldots, i-1: \operatorname{Right}_{f, K} \\
\text { Key } i \text { : PuncOutputZ } f_{i}, K_{i}^{\prime}, s^{*} \\
\text { Keys } i+1, \ldots, T_{1} \text { : } \text { Left }_{f, K}\end{array}$ & $\begin{array}{l}\text { PuncOutputY }_{f, K^{\prime}, y, s^{*}} \\
\qquad y \leftarrow f\left(x_{1}^{*}\right)\end{array}$ \\
\hline $\mathcal{H}^{(10, i)}$ & $\begin{array}{c}\left(x_{b}^{*}, x_{1}^{*}, s^{*}, z^{*}\right) \\
z^{*}=f_{i}\left(x_{1}^{*} ; \operatorname{PRF}_{K_{i}}\left(s^{*}\right)\right)\end{array}$ & $\begin{array}{l}\text { Keys } 1, \ldots, i \text { : Right } \\
\text { Keys } i+1, \ldots, T_{1} \text { : } \text { Left }_{f, K}\end{array}$ & $\begin{array}{c}\text { PuncOutput }_{f, K^{\prime}, y, s^{*}} \\
y \leftarrow f\left(x_{1}^{*}\right)\end{array}$ \\
\hline $\mathcal{H}^{(11)}$ & $\left(x_{1}^{*}, x_{1}^{*}, s^{*}, \perp\right)$ & Right $_{f, K}$ & $\begin{array}{c}\text { PuncOutput }_{f, K^{\prime}, y, s^{*}} \\
y \leftarrow f\left(x_{1}^{*}\right)\end{array}$ \\
\hline
\end{tabular}

Table 2: The differences between the experiments $\mathcal{H}^{(0)}, \ldots, \mathcal{H}^{(11)}$. 


\section{PuncOutput $_{f, K^{\prime}, y, s^{*}}\left(x_{L}, x_{R}, s, z\right)$ :}

1. If $s=s^{*}$ then output $y$.

2. Otherwise, let $r=\operatorname{PRF}$.Eval $\left(K^{\prime}, s\right)$ and output $f\left(x_{L} ; r\right)$.

\section{PuncOutputZ $Z_{f, K^{\prime}, s^{*}}\left(x_{L}, x_{R}, s, z\right)$ :}

1. If $s=s^{*}$ then output $z$.

2. Otherwise, let $r=$ PRF.Eval $\left(K^{\prime}, s\right)$ and output $f\left(x_{L} ; r\right)$.

Figure 3: The functions PuncOutput $Y_{f, K^{\prime}, y, s^{*}}$ and PuncOutput $Z_{f, K^{\prime}, s^{*}}$.

Note that for each function $f \in\left\{f_{1}, \ldots, f_{T}\right\}$ with an associated PRF key $K$, for the deterministic function Left $f_{f, K}$ and the challenge ciphertext it holds that $\operatorname{Left}_{f, K}\left(x_{b}^{*}, x_{1}^{*}, s^{*}, \perp\right)=\operatorname{Left}_{f, K}\left(x_{b}^{*}, \perp, s^{*}\right.$, $\perp$ ). Therefore, the message privacy of the underlying scheme FE (with respect to deterministic functions) guarantees that the adversary $\mathcal{A}$ has only a negligible advantage in distinguishing experiments $\mathcal{H}^{(0)}$ and $\mathcal{H}^{(1)}$. Specifically, let $\mathcal{F}^{\prime}$ denote the family of deterministic functions Left $f, K$ and Right $_{f, K}$ for every $f \in \mathcal{F}$ and PRF key $K$ (as defined in Figure 1) as well as the function PuncOutput $Y_{f, K^{\prime}, y, s^{*}}$ and PuncOutputZ $Z_{f, K^{\prime}, s^{*}}$ for every $f \in \mathcal{F}$, punctured PRF key $K^{\prime}$, value $y \in \mathcal{Y}_{\lambda}$ and string $s^{*} \in\{0,1\}^{\lambda}$ (as defined in Figure 3). In Appendix $\mathrm{C}$ we prove the following claim:

Claim 4.12. There exists a probabilistic polynomial-time adversary $\mathcal{B}^{(0) \rightarrow(1)}$ such that

$$
\left|\operatorname{Pr}\left[\mathcal{H}^{(0)}(\lambda)=1\right]-\operatorname{Pr}\left[\mathcal{H}^{(1)}(\lambda)=1\right]\right| \leq \operatorname{Adv}_{\mathrm{FE}, \mathcal{F}^{\prime}, \mathcal{B}(0) \rightarrow(1), T}^{\mathrm{MP}}(\lambda)
$$

Experiment $\mathcal{H}^{(2, i)}(\lambda)$ where $i \in\left[\boldsymbol{T}_{2}+1\right]$. This experiment is obtained from the experiment $\mathcal{H}^{(1)}(\lambda)$ by modifying the post challenge key-generation oracle to generate keys as follows. The functional keys for the $f_{T_{1}+1}, \ldots, f_{T_{1}+i-1}$ are generated as PuncOutput $Y_{f, K^{\prime}, y, s^{*}}$ (see Figure 3 for the definition of PuncOutput $Y_{f, K^{\prime}, y, s^{*}}$, where $K^{\prime}$ is generated by sampling a PRF key $K \leftarrow$ PRF.Gen $\left(1^{\lambda}\right)$ and then puncturing it at $s^{*}$, and where $y \leftarrow f\left(x_{b}^{*}\right)$, and the functional keys for $f_{T_{1}+i}, \ldots, f_{T_{1}+T_{2}}=f_{T}$ are generated as PuncOutputY $Y_{f, K^{\prime}, y, s^{*}}$, where $K^{\prime}$ and $s^{*}$ are as before but $y=f\left(x_{b}^{*} ; \operatorname{PRF}_{K}\left(s^{*}\right)\right)$.

Note that every $x \neq x_{b}^{*}$ with which the encryption oracle is queries (with probability negligibly close to 1) it holds that $s \neq s^{*}$, hence, using the functionality feature of the punctured PRF, for every $f \in\left\{f_{T_{1}+1}, \ldots, f_{T}\right\}$ it holds that $\operatorname{Left}_{f, K}(x, x, s, \perp)=$ PuncOutputY $_{f, K^{\prime}, y, s *}(x, x, s, \perp)$. In addition, for the challenge $x_{b}^{*}$ it holds that $\operatorname{Left}_{f, K}\left(x_{b}^{*}, x_{1}^{*}, s^{*}, \perp\right)=\operatorname{PuncOutput}_{f, K^{\prime}, y, s *}\left(x_{b}^{*}, x_{1}^{*}, s^{*}, \perp\right)$ since PuncOutputY $Y_{f, K^{\prime}, y, s *}$ simply outputs $y$, where $y=f\left(x_{b}^{*} ; \operatorname{PRF}_{K}\left(s^{*}\right)\right)$. Thus, the function-privacy of the underlying scheme FE guarantees that the adversary $\mathcal{A}$ has only a negligible advantage in distinguishing experiments $\mathcal{H}^{(1)}(\lambda)$ and $\mathcal{H}^{(2,1)}(\lambda)$. In Appendix $\mathrm{C}$ we prove the following claim:

Claim 4.13. There exists a probabilistic polynomial-time adversary $\mathcal{B}^{(1) \rightarrow(2,1)}$ such that

$$
\left|\operatorname{Pr}\left[\mathcal{H}^{(1)}(\lambda)=1\right]-\operatorname{Pr}\left[\mathcal{H}^{(2,1)}(\lambda)=1\right]\right| \leq \operatorname{Adv}_{\mathrm{FE}, \mathcal{F}^{\prime}, \mathcal{B}^{(1) \rightarrow(2,1)}, T}^{\mathrm{FP}}(\lambda)+\operatorname{neg}(\lambda) .
$$

Moreover, note that the pseudorandomness of $\operatorname{PRF}_{K}(\cdot)$ at punctured point $s^{*}$ (see Definition 2.2) guarantees that the adversary $\mathcal{A}$ has only a negligible advantage in distinguishing experiments $\mathcal{H}^{(2, i)}$ and $\mathcal{H}^{(2, i+1)}$. In Appendix $\mathrm{C}$ we prove the following claim:

Claim 4.14. For every $i \in\left[T_{2}\right]$ there exists a probabilistic polynomial-time adversary $\mathcal{B}^{(2, i) \rightarrow(2, i+1)}$ such that

$$
\left|\operatorname{Pr}\left[\mathcal{H}^{(2, i)}(\lambda)=1\right]-\operatorname{Pr}\left[\mathcal{H}^{(2, i+1)}(\lambda)=1\right]\right| \leq \operatorname{Adv}_{\text {puPRF }, \mathcal{B}^{(2, i) \rightarrow(2, i+1)}}(\lambda) .
$$


Experiment $\mathcal{H}^{(3, i)}(\boldsymbol{\lambda})$ where $i \in\left[\boldsymbol{T}_{\mathbf{2}}+\mathbf{1}\right]$. This experiment is obtained from the experiment $\mathcal{H}^{\left(2, T_{2}\right)}(\lambda)$ by modifying the post-challenge key-generation oracle as follows. The functional keys for the $f_{T_{1}+1}, \ldots, f_{T_{1}+i-1}$ are generated as PuncOutput $Y_{f, K^{\prime}, y, s^{*}}$, where $K^{\prime}$ is generated by sampling a PRF key $K \leftarrow$ PRF.Gen $\left(1^{\lambda}\right)$ and then puncturing it at $s^{*}$, and where $y \leftarrow f\left(x_{1}^{*}\right)$, and the functional keys for $f_{T_{1}+i}, \ldots, f_{T_{1}+T_{2}}$ are generated as PuncOutput $\mathrm{Y}_{f, K^{\prime}, y, s^{*}}$, where $K^{\prime}$ and $s^{*}$ are as before but $y \leftarrow f\left(x_{b}^{*}\right)$. We observe that $\mathcal{H}^{(2, T+1)}(\lambda)=\mathcal{H}^{(3,1)}(\lambda)$.

The adaptive admissibility of the function family $\mathcal{F}$ (see Definition 3.4) guarantee that the advantage of the adversary $\mathcal{A}$ in distinguishing experiments $\mathcal{H}^{(3, i)}$ and $\mathcal{H}^{(3, i+1)}$ is at most $\Delta(\lambda)$. In Appendix $\mathrm{C}$ we prove the following claim:

Claim 4.15. For every $i \in\left[T_{2}\right]$ there exists a probabilistic polynomial-time adversary $\mathcal{B}^{(3, i) \rightarrow(3, i+1)}$ such that

$$
\left|\operatorname{Pr}\left[\mathcal{H}^{(3, i)}(\lambda)=1\right]-\operatorname{Pr}\left[\mathcal{H}^{(3, i+1)}(\lambda)=1\right]\right| \leq \operatorname{Adv}_{\mathcal{F}, \mathcal{B}^{(3, i) \rightarrow(3, i+1)}}^{\mathrm{aADM}} \leq \Delta(\lambda)
$$

Experiment $\mathcal{H}^{(4, i)}(\lambda)$ where $i \in\left[\boldsymbol{T}_{\mathbf{1}}+\mathbf{1}\right]$. This experiment is obtained from the experiment $\mathcal{H}^{(3, T)}(\lambda)$ by modifying the pre-challenge key-generation oracle as follows. The functional keys for $f_{1}, \ldots, f_{i-1}$ are generated as sk $\leftarrow$ FE.KG(msk, Right $\left._{f, K}\right)$ instead of as sk $f \leftarrow$ FE.KG $\left(\right.$ msk, $_{f}$ Left $\left._{f, K}\right)$ (where Right $f_{f, K}$ is defined in Figure 1), and the functional keys for $f_{i}, \ldots, f_{T_{1}}$ are generated as before (i.e., as sk $f \leftarrow$ FE.KG $\left(\right.$ msk, Left $\left.f_{, K}\right)$ ). We observe that $\mathcal{H}^{(3, T+1)}(\lambda)=\mathcal{H}^{(4,1)}(\lambda)$.

Experiment $\mathcal{H}^{(5, i)}(\lambda)$ where $i \in\left[\boldsymbol{T}_{\mathbf{1}}\right]$. This experiment is obtained from the experiment $\mathcal{H}^{(4, i)}(\lambda)$ by modifying the encryption oracle so that on the challenge input $\left(x_{0}^{*}, x_{1}^{*}\right)$ it samples $s^{*} \leftarrow\{0,1\}^{\lambda}$ and outputs ct $\leftarrow$ FE.Enc $\left(\operatorname{msk},\left(x_{b}^{*}, x_{1}^{*}, s^{*}, z^{*}\right)\right)$, where $z^{*}=f_{i}\left(x_{b}^{*}\right.$; PRF.Eval $\left.\left(K_{i}, s^{*}\right)\right)$, instead of ct $\leftarrow$ FE. Enc $\left(\operatorname{msk},\left(x_{b}^{*}, x_{1}^{*}, s^{*}, \perp\right)\right)$.

Notice that both Left $f, K$ and Right ${ }_{f, K}$ are defined to ignore the fourth input $z$, hence, for the first $i-1$ keys it holds that $\operatorname{Right}_{f, K}\left(x_{b}^{*}, x_{1}^{*}, s^{*}, \perp\right)=\operatorname{Right}_{f, K}\left(x_{b}^{*}, x_{1}^{*}, s^{*}, z^{*}\right)$ and for the next $T_{1}-i+1$ keys it holds that $\operatorname{Left}_{f, K}\left(x_{b}^{*}, x_{1}^{*}, s^{*}, \perp\right)=\operatorname{Left}_{f, K}\left(x_{b}^{*}, x_{1}^{*}, s^{*}, z^{*}\right)$. Therefore, the message privacy of the underlying scheme FE guarantees that the adversary $\mathcal{A}$ has only a negligible advantage in distinguishing experiments $\mathcal{H}^{(4, i)}$ and $\mathcal{H}^{(5, i)}$. In Appendix $\mathrm{C}$ we prove the following claim:

Claim 4.16. For every $i \in\left[T_{1}\right]$ there exists a probabilistic polynomial-time adversary $\mathcal{B}^{(4, i) \rightarrow(5, i)}$ such that

$$
\left|\operatorname{Pr}\left[\mathcal{H}^{(4, i)}(\lambda)=1\right]-\operatorname{Pr}\left[\mathcal{H}^{(5, i)}(\lambda)=1\right]\right| \leq \operatorname{Adv}_{\mathrm{FE}, \mathcal{F}^{\prime}, \mathcal{B}^{(4, i) \rightarrow(5, i)}, T}(\lambda) .
$$

Experiment $\mathcal{H}^{(6, i)}(\boldsymbol{\lambda})$ where $\boldsymbol{i} \in\left[\boldsymbol{T}_{\mathbf{1}}\right]$. This experiment is obtained from the experiment $\mathcal{H}^{(5, i)}(\lambda)$ by modifying the behavior of the pre-challenge key-generation oracle on the $i$ th query $f_{i}$ (without modifying its behavior on all other queries). On input the $i$ th query $f_{i}$, the pre-challenge keygeneration oracle compute $\mathrm{sk}_{f_{i}} \leftarrow$ FE.KG(msk, PuncOutputZ $\left.f_{f_{i}, K_{i}^{\prime}, s^{*}}\right)$ instead of $\mathrm{sk}_{f_{i}} \leftarrow \mathrm{FE} . \mathrm{KG}(\mathrm{msk}$, Left $_{f_{i}, K_{i}}$ ) (where the function PuncOutputZ $Z_{f_{i}, K_{i}^{\prime}, s^{*}}$ is defined in Figure 3).

Note that by the functionality feature of the punctured PRF (see Definition 2.2), for every ciphertext $(x, \perp, s, z)$ which is not the challenge ciphertext (with probability negligibly close to 1 ) it holds that PuncOutputZ $f_{i}, K_{i}^{\prime}, s^{*}(x, \perp, s, z)=\operatorname{Left}_{f_{i}, K_{i}}(x, \perp, s, z)$ (since $s \neq s^{*}$ with very high probability). For the challenge ciphertext the latter also holds since PuncOutputZ $\mathrm{f}_{i}, K_{i}^{\prime}, s^{*}\left(x_{b}^{*}, x_{1}^{*}, s^{*}, z^{*}\right)$ outputs $z^{*}=f_{i}\left(x_{b}^{*} ; \operatorname{PRF}_{K_{i}}\left(s^{*}\right)\right)$. Thus, the function-privacy of the underlying scheme FE guarantees that the adversary $\mathcal{A}$ has only a negligible advantage in distinguishing experiments $\mathcal{H}^{(6, i)}(\lambda)$ and $\mathcal{H}^{(7, i)}(\lambda)$. In Appendix $\mathrm{C}$ we prove the following claim: 
Claim 4.17. For every $i \in\left[T_{1}\right]$ there exists a probabilistic polynomial-time adversary $\mathcal{B}^{(5, i) \rightarrow(6, i)}$ such that

$$
\left|\operatorname{Pr}\left[\mathcal{H}^{(5, i)}(\lambda)=1\right]-\operatorname{Pr}\left[\mathcal{H}^{(6, i)}(\lambda)=1\right]\right| \leq \operatorname{Adv}_{\mathrm{FE}, \mathcal{F}^{\prime}, \mathcal{B}^{(5, i) \rightarrow(6, i)}, T}^{\mathrm{FP}}(\lambda)+\operatorname{neg}(\lambda) .
$$

Experiment $\mathcal{H}^{(7, i)}(\boldsymbol{\lambda})$ where $\boldsymbol{i} \in\left[\boldsymbol{T}_{\mathbf{1}}\right]$. This experiment is obtained from the experiment $\mathcal{H}^{(6, i)}(\lambda)$ by modifying the encryption oracle so that on the challenge input $\left(x_{0}^{*}, x_{1}^{*}\right)$ it outputs ct $\leftarrow$ FE.Enc $(\mathrm{msk}$, $\left.\left(x_{b}^{*}, x_{1}^{*}, s^{*}, z^{*}\right)\right)$, where $z^{*}=f_{i}\left(x_{b}^{*} ; r^{*}\right)$ for a fresh and uniformly sampled value $r^{*}$ instead of $z^{*}=f_{i}\left(x_{b}^{*} ; \operatorname{PRF} . \operatorname{Eval}\left(K_{i}, s^{*}\right)\right)$.

The pseudorandomness at punctured point $s^{*}$ of PRF.Eval $\left(K_{i}, \cdot\right)$ guarantees that the adversary $\mathcal{A}$ has only a negligible advantage in distinguishing experiments $\mathcal{H}^{(6, i)}$ and $\mathcal{H}^{(7, i)}$. In Appendix $\mathrm{C}$ we prove the following claim:

Claim 4.18. For every $i \in\left[T_{1}\right]$ there exists a probabilistic polynomial-time adversary $\mathcal{B}^{(6, i) \rightarrow(7, i)}$ such that

$$
\left|\operatorname{Pr}\left[\mathcal{H}^{(6, i)}(\lambda)=1\right]-\operatorname{Pr}\left[\mathcal{H}^{(7, i)}(\lambda)=1\right]\right| \leq \operatorname{Adv}_{\text {puPRF }, \mathcal{B}^{(6, i) \rightarrow(7, i)}}(\lambda) .
$$

Experiment $\mathcal{H}^{(8, i)}(\boldsymbol{\lambda})$ where $\boldsymbol{i} \in\left[\boldsymbol{T}_{\mathbf{1}}\right]$. This experiment is obtained from the experiment $\mathcal{H}^{(7, i)}(\lambda)$ by modifying the encryption oracle so that on the challenge input $\left(x_{0}^{*}, x_{1}^{*}\right)$ it outputs ct $\leftarrow \mathrm{FE}$.Enc $(\mathrm{msk}$, $\left.\left(x_{b}^{*}, x_{1}^{*}, s^{*}, z^{*}\right)\right)$, where $z^{*}=f_{i}\left(x_{1}^{*} ; r^{*}\right)$ instead of $z^{*}=f_{i}\left(x_{b}^{*} ; r^{*}\right)$ (both with fresh and uniform $\left.r^{*}\right)$.

The adaptive admissibility of the function family $\mathcal{F}$ (see Definition 3.4) guarantees that the advantage of the adversary $\mathcal{A}$ in distinguishing experiments $\mathcal{H}^{(7, i)}$ and $\mathcal{H}^{(8, i)}$ is at most $\Delta(\lambda)$. In Appendix $\mathrm{C}$ we prove the following claim:

Claim 4.19. For every $i \in\left[T_{1}\right]$ there exists a probabilistic polynomial-time adversary $\mathcal{B}^{(7, i) \rightarrow(8, i)}$ such that

$$
\left|\operatorname{Pr}\left[\mathcal{H}^{(7, i)}(\lambda)=1\right]-\operatorname{Pr}\left[\mathcal{H}^{(8, i)}(\lambda)=1\right]\right| \leq \operatorname{Adv}_{\mathcal{F}, \mathcal{B}^{(7, i) \rightarrow(8, i)}}^{\mathrm{aADM}} \leq \Delta(\lambda) .
$$

Experiment $\mathcal{H}^{(9, i)}(\lambda)$ where $\boldsymbol{i} \in\left[\boldsymbol{T}_{\mathbf{1}}\right]$. This experiment is obtained from the experiment $\mathcal{H}^{(8, i)}(\lambda)$ by modifying the encryption oracle so that on the challenge input $\left(x_{0}^{*}, x_{1}^{*}\right)$ it outputs ct $\leftarrow \mathrm{FE}$.Enc $(\mathrm{msk}$, $\left.\left(x_{b}^{*}, x_{1}^{*}, s^{*}, z^{*}\right)\right)$, where $z^{*}=f_{i}\left(x_{1}^{*} ; \operatorname{PRF} . \operatorname{Eval}\left(K_{i}, s^{*}\right)\right)$ instead of $z^{*}=f_{i}\left(x_{1}^{*} ; r^{*}\right)$ for a fresh and uniformly sampled value $r^{*}$.

The pseudorandomness at punctured point $s^{*}$ of PRF.Eval $\left(K_{i}, \cdot\right)$ guarantees that the adversary $\mathcal{A}$ has only a negligible advantage in distinguishing experiments $\mathcal{H}^{(9, i)}$ and $\mathcal{H}^{(10, i)}$. The proof of the following claim is essentially identical to the proof of Claim 4.18 (see Appendix C):

Claim 4.20. For every $i \in\left[T_{1}\right]$ there exists a probabilistic polynomial-time adversary $\mathcal{B}^{(8, i) \rightarrow(9, i)}$ such that

$$
\left|\operatorname{Pr}\left[\mathcal{H}^{(8, i)}(\lambda)=1\right]-\operatorname{Pr}\left[\mathcal{H}^{(9, i)}(\lambda)=1\right]\right| \leq \operatorname{Adv}_{\text {puPRF }, \mathcal{B}^{(8, i) \rightarrow(9, i)}}(\lambda) .
$$

Experiment $\mathcal{H}^{(\mathbf{1 0}, \boldsymbol{i})}(\boldsymbol{\lambda})$ where $\boldsymbol{i} \in\left[\boldsymbol{T}_{\mathbf{1}}\right]$. This experiment is obtained from the experiment $\mathcal{H}^{(9, i)}(\lambda)$ by modifying the behavior of the pre-challenge key-generation oracle on the $i$ th query $f_{i}$ (without modifying its behavior on all other queries). On input the $i$ th query $f_{i}$, the key-generation oracle compute $\mathrm{sk}_{f_{i}} \leftarrow$ FE.KG (msk, Right $\left._{f_{i}, K_{i}}\right)$ instead of sk $f_{i} \leftarrow$ FE.KG(msk, PuncOutputZ $\left.f_{f_{i}, K_{i}^{\prime}, s^{*}}\right)$. 
As in the proof of Claim 4.8, the selective-function function privacy of the underlying scheme FE (with respect to deterministic functions) guarantees that the adversary $\mathcal{A}$ has only a negligible advantage in distinguishing experiments $\mathcal{H}^{(9, i)}$ and $\mathcal{H}^{(10, i)}$. The proof of the following claim is essentially identical to the proof of Claim 4.17 (see Appendix C):

Claim 4.21. For every $i \in\left[T_{1}\right]$ there exists a probabilistic polynomial-time adversary $\mathcal{B}^{(9, i) \rightarrow(10, i)}$ such that

$$
\left|\operatorname{Pr}\left[\mathcal{H}^{(9, i)}(\lambda)=1\right]-\operatorname{Pr}\left[\mathcal{H}^{(10, i)}(\lambda)=1\right]\right| \leq \operatorname{Adv}_{\mathrm{FE}, \mathcal{F}^{\prime}, \mathcal{B}^{(9, i) \rightarrow(10, i), T}}^{\mathrm{FP}}(\lambda)+\operatorname{neg}(\lambda) .
$$

Next, we observe that experiment $\mathcal{H}^{(4, i+1)}(\lambda)$ is obtained from the experiment $\mathcal{H}^{(10, i)}(\lambda)$ by modifying the challenge ciphertext to be computed using $z^{*}=\perp$ instead of $z^{*}=f_{i}\left(x_{1}^{*}\right.$; PRF.Eval $\left.\left(K_{i}, s^{*}\right)\right)$.

Note that for each function $f \in\left\{f_{1}, \ldots, f_{T}\right\}$ with an associated PRF key $K$, for the deterministic functions Left $f_{f, K}$ and Right $_{f, K}$ and the challenge ciphertext it holds that $\operatorname{Left}_{f, K}\left(x_{b}^{*}, x_{1}^{*}, s^{*}, \perp\right)=$ Left $_{f, K}\left(x_{b}^{*}, x_{1}^{*}, s^{*}, z^{*}\right)$ and Right ${ }_{f, K}\left(x_{b}^{*}, x_{1}^{*}, s^{*}, \perp\right)=\operatorname{Right}_{f, K}\left(x_{b}^{*}, x_{1}^{*}, s^{*}, z^{*}\right)$. Therefore, the selectivefunction message privacy of the underlying scheme FE (with respect to deterministic functions) guarantees that the adversary $\mathcal{A}$ has only a negligible advantage in distinguishing experiments $\mathcal{H}^{(10, i)}$ and $\mathcal{H}^{(4, i+1)}$. The proof of the following claim is essentially identical to the proof of Claim 4.16 (see Appendix C):

Claim 4.22. For every $i \in\left[T_{1}\right]$ there exists a probabilistic polynomial-time adversary $\mathcal{B}^{(10, i) \rightarrow(4, i+1)}$ such that

$$
\left|\operatorname{Pr}\left[\mathcal{H}^{(10, i)}(\lambda)=1\right]-\operatorname{Pr}\left[\mathcal{H}^{(4, i+1)}(\lambda)=1\right]\right| \leq \operatorname{Adv}_{\mathrm{FE}, \mathcal{F}^{\prime}, \mathcal{B}^{(10, i) \rightarrow(4, i+1)}, T}(\lambda)
$$

Experiment $\mathcal{H}^{(\mathbf{1 1})}(\boldsymbol{\lambda})$. This experiment is obtained from the experiment $\mathcal{H}^{(4, T+1)}(\lambda)$ by modifying the encryption oracle so that on the challenge input $\left(x_{0}^{*}, x_{1}^{*}\right)$ it outputs ct $\leftarrow \mathrm{FE}$.Enc(msk, $\left.\left(x_{1}^{*}, x_{1}^{*}, s^{*}, \perp\right)\right)$ instead of ct $\leftarrow$ FE.Enc $\left(\mathrm{msk},\left(x_{b}^{*}, x_{1}^{*}, s^{*}, \perp\right)\right)$. Note that this experiment is completely independent of the bit $b$, and therefore $\operatorname{Pr}\left[\mathcal{H}^{(11)}(\lambda)=1\right]=1 / 2$.

In addition, note that for every function $f \in\left\{f_{1}, \ldots, f_{T_{1}}\right\}$ with an associated PRF key $K$, for the deterministic function $\operatorname{Right}_{f, K}$ it holds that $\operatorname{Right}_{f, K}\left(x_{b}^{*}, x_{1}^{*}, s^{*}, \perp\right)=\operatorname{Right}_{f, K}\left(x_{1}^{*}, x_{1}^{*}, s^{*}, \perp\right)$. Therefore, the message privacy of the underlying scheme FE (with respect to deterministic functions) guarantees that the adversary $\mathcal{A}$ has only a negligible advantage in distinguishing experiments $\mathcal{H}^{\left(4, T_{1}+1\right)}$ and $\mathcal{H}^{(11)}$. The proof of the following claim is essentially identical to the proof of Claim 4.12 (see Appendix C):

Claim 4.23. There exists a probabilistic polynomial-time adversary $\mathcal{B}^{\left(4, T_{1}+1\right) \rightarrow(11)}$ such that

$$
\left|\operatorname{Pr}\left[\mathcal{H}^{\left(4, T_{1}+1\right)}(\lambda)=1\right]-\operatorname{Pr}\left[\mathcal{H}^{(11)}(\lambda)=1\right]\right| \leq \operatorname{Adv}_{\mathrm{FE}, \mathcal{F}^{\prime}, \mathcal{B}^{(4, T+1) \rightarrow(11), T}}^{\mathrm{sfFP}}(\lambda)
$$

Finally, putting together Claims 4.12-4.23 with the facts that $\operatorname{Expt}_{\Pi, \mathcal{F}, \mathcal{A}, T}^{\mathrm{aMPRF}}(\lambda)=\mathcal{H}^{(0)}(\lambda), \mathcal{H}^{(1)}(\lambda)$ $=\mathcal{H}^{(2,1)}(\lambda), \mathcal{H}^{(2, T+1)}(\lambda)=\mathcal{H}^{(3,1)}(\lambda), \mathcal{H}^{(3, T+1)}(\lambda)=\mathcal{H}^{(4,1)}(\lambda)$ and $\operatorname{Pr}\left[\mathcal{H}^{(11)}(\lambda)=1\right]=1 / 2$, we 
observe that

$$
\begin{aligned}
\operatorname{Adv}_{\Pi, \mathcal{F}, \mathcal{A}, T}^{\mathrm{aMPRF}}= & \left|\operatorname{Pr}\left[\operatorname{Expt}_{\Pi, \mathcal{F}, \mathcal{A}, T}^{\mathrm{aMPRF}}(\lambda)=1\right]-\frac{1}{2}\right| \\
= & \left|\operatorname{Pr}\left[\mathcal{H}^{(0)}(\lambda)=1\right]-\operatorname{Pr}\left[\mathcal{H}^{(11)}(\lambda)=1\right]\right| \\
\leq & \left|\operatorname{Pr}\left[\mathcal{H}^{(0)}(\lambda)=1\right]-\operatorname{Pr}\left[\mathcal{H}^{(1)}(\lambda)=1\right]\right| \\
& +\left|\operatorname{Pr}\left[\mathcal{H}^{(1)}(\lambda)=1\right]-\operatorname{Pr}\left[\mathcal{H}^{(2,1)}(\lambda)=1\right]\right| \\
& +\sum_{j=2}^{3} \sum_{i=1}^{T_{2}}\left|\operatorname{Pr}\left[\mathcal{H}^{(j, i)}(\lambda)=1\right]-\operatorname{Pr}\left[\mathcal{H}^{(j, i+1)}(\lambda)=1\right]\right| \\
& +\sum_{i=1}^{T_{1}} \sum_{j=4}^{9}\left|\operatorname{Pr}\left[\mathcal{H}^{(j, i)}(\lambda)=1\right]-\operatorname{Pr}\left[\mathcal{H}^{(j+1, i)}(\lambda)=1\right]\right| \\
& +\sum_{i=1}^{T_{1}}\left|\operatorname{Pr}\left[\mathcal{H}^{(10, i)}(\lambda)=1\right]-\operatorname{Pr}\left[\mathcal{H}^{(4, i+1)}(\lambda)=1\right]\right| \\
& +\left|\operatorname{Pr}\left[\mathcal{H}^{(4, T+1)}(\lambda)=1\right]-\operatorname{Pr}\left[\mathcal{H}^{(11)}(\lambda)=1\right]\right| \\
\leq & \left(T_{1}(\lambda)+T_{2}(\lambda)\right) \cdot \Delta(\lambda)+\operatorname{neg}(\lambda) \\
= & T(\lambda) \cdot \Delta(\lambda)+\operatorname{neg}(\lambda) .
\end{aligned}
$$

\section{Acknowledgments}

We thank Zvika Brakerski for various insightful discussions.

\section{References}

$\left[\mathrm{AAB}^{+} 13\right]$ S. Agrawal, S. Agrawal, S. Badrinarayanan, A. Kumarasubramanian, M. Prabhakaran, and A. Sahai. Function private functional encryption and property preserving encryption: New definitions and positive results. Cryptology ePrint Archive, Report 2013/744, 2013.

$\left[\mathrm{ABG}^{+} 13\right]$ P. Ananth, D. Boneh, S. Garg, A. Sahai, and M. Zhandry. Differing-inputs obfuscation and applications. Cryptology ePrint Archive, Report 2013/689, 2013.

$\left[\mathrm{AGV}^{+} 13\right]$ S. Agrawal, S. Gorbunov, V. Vaikuntanathan, and H. Wee. Functional encryption: New perspectives and lower bounds. In Advances in Cryptology - CRYPTO '13, pages 500$518,2013$.

[BCP14] E. Boyle, K. Chung, and R. Pass. On extractability obfuscation. In Proceedings of the 11th Theory of Cryptography Conference, pages 52-73, 2014.

[BF03] D. Boneh and M. K. Franklin. Identity-based encryption from the Weil pairing. SIAM Journal on Computing, 32(3):586-615, 2003. Preliminary version in Advances in Cryptology - CRYPTO '01, pages 213-229, 2001. 
[BGI $\left.{ }^{+} 12\right]$ B. Barak, O. Goldreich, R. Impagliazzo, S. Rudich, A. Sahai, S. P. Vadhan, and K. Yang. On the (im)possibility of obfuscating programs. Journal of the ACM, 59(2):6, 2012.

[BGI14] E. Boyle, S. Goldwasser, and I. Ivan. Functional signatures and pseudorandom functions. In Proceedings of the 17th International Conference on Practice and Theory in PublicKey Cryptography, pages 501-519, 2014.

[BO13] M. Bellare and A. O'Neill. Semantically-secure functional encryption: Possibility results, impossibility results and the quest for a general definition. In Proceedings of the 12th International Conference on Cryptology and Network Security, pages 218-234, 2013.

[BRS13a] D. Boneh, A. Raghunathan, and G. Segev. Function-private identity-based encryption: Hiding the function in functional encryption. In Advances in Cryptology - CRYPTO '13, pages 461-478, 2013.

[BRS13b] D. Boneh, A. Raghunathan, and G. Segev. Function-private subspace-membership encryption and its applications. In Advances in Cryptology - ASIACRYPT '13, pages 255-275, 2013.

[BS14] Z. Brakerski and G. Segev. Function-private functional encryption in the private-key setting. Cryptology ePrint Archive, Report 2014/550, 2014.

[BSW11] D. Boneh, A. Sahai, and B. Waters. Functional encryption: Definitions and challenges. In Proceedings of the 8th Theory of Cryptography Conference, pages 253-273, 2011.

[BW13] D. Boneh and B. Waters. Constrained pseudorandom functions and their applications. In Advances in Cryptology - ASIACRYPT '13, pages 280-300, 2013.

[Coc01] C. Cocks. An identity based encryption scheme based on quadratic residues. In Proceedings of the 8th IMA International Conference on Cryptography and Coding, pages 360-363, 2001.

$\left[\mathrm{DIJ}^{+}{ }^{13}\right.$ A. De Caro, V. Iovino, A. Jain, A. O’Neill, O. Paneth, and G. Persiano. On the achievability of simulation-based security for functional encryption. In Advances in Cryptology - CRYPTO '13, pages 519-535, 2013.

[GGG $\left.{ }^{+} 14\right]$ S. Goldwasser, S. D. Gordon, V. Goyal, A. Jain, J. Katz, F.-H. Liu, A. Sahai, E. Shi, and H.-S. Zhou. Multi-input functional encryption. In Advances in Cryptology - EUROCRYPT'14, pages 578-602, 2014.

$\left[\mathrm{GGH}^{+} 13\right]$ S. Garg, C. Gentry, S. Halevi, M. Raykova, A. Sahai, and B. Waters. Candidate indistinguishability obfuscation and functional encryption for all circuits. In Proceedings of the 54th Annual IEEE Symposium on Foundations of Computer Science, pages 40-49, 2013.

$\left[\mathrm{GGH}^{+} 14\right]$ S. Garg, C. Gentry, S. Halevi, and M. Zhandry. Fully secure functional encryption without obfuscation. Cryptology ePrint Archive, Report 2014/666, 2014.

[GGM86] O. Goldreich, S. Goldwasser, and S. Micali. How to construct random functions. Journal of the ACM, 33(4):792-807, 1986.

[GJK $\left.{ }^{+} 13\right]$ V. Goyal, A. Jain, V. Koppula, and A. Sahai. Functional encryption for randomized functionalities. Cryptology ePrint Archive, Report 2013/729, 2013. 
$\left[\mathrm{GKP}^{+} 13\right]$ S. Goldwasser, Y. Kalai, R. A. Popa, V. Vaikuntanathan, and N. Zeldovich. Reusable garbled circuits and succinct functional encryption. In Proceedings of the 45th Annual ACM Symposium on Theory of Computing, pages 555-564, 2013.

[GVW12] S. Gorbunov, V. Vaikuntanathan, and H. Wee. Functional encryption with bounded collusions via multi-party computation. In Advances in Cryptology - CRYPTO '12, pages 162-179, 2012.

$\left[\mathrm{KPT}^{+} 13\right]$ A. Kiayias, S. Papadopoulos, N. Triandopoulos, and T. Zacharias. Delegatable pseudorandom functions and applications. In Proceedings of the 20th Annual ACM Conference on Computer and Communications Security, pages 669-684, 2013.

[O'N10] A. O'Neill. Definitional issues in functional encryption. Cryptology ePrint Archive, Report 2010/556, 2010.

[Sha84] A. Shamir. Identity-based cryptosystems and signature schemes. In Advances in Cryptology - CRYPTO '84, pages 47-53, 1984.

[SSW09] E. Shen, E. Shi, and B. Waters. Predicate privacy in encryption systems. In Proceedings of the 6th Theory of Cryptography Conference, pages 457-473, 2009.

[SW08] A. Sahai and B. Waters. Slides on functional encryption. Available at http://www.cs . utexas.edu/ bwaters/presentations/files/functional.ppt, 2008.

[SW14] A. Sahai and B. Waters. How to use indistinguishability obfuscation: deniable encryption, and more. In Proceedings of the 46th Annual ACM Symposium on Theory of Computing, pages 475-484, 2014.

[Wat14] B. Waters. A punctured programming approach to adaptively secure functional encryption. Cryptology ePrint Archive, Report 2014/588, 2014.

\section{A A Comparison with the Definitional Framework of Goyal et al. [GJK $\left.{ }^{+} 13\right]$}

Our approach for defining message privacy is inspired by that of Goyal et al. but there are a few subtle differences between the two approaches. As mentioned in Section 1.3, as far as we are able to currently tell, it seems that both our scheme and the scheme of Goyal et al. provide message privacy according to both of these approaches. We emphasize once again that we view the main contribution of our paper as basing the security of our scheme on any underlying functional encryption scheme (and avoiding obfuscation-related assumptions), and not as offering alternative notions of message privacy. In what follows we point out a couple of differences between the two approaches ${ }^{7}$.

- Goyal et al. allow adversaries to obtain functional keys only for functions $f$ for which the distributions $f\left(x_{0}^{*}\right)$ and $f\left(x_{1}^{*}\right)$ are negligibly close (either computationally or statistically), whereas our framework does not include such a restriction. In our framework, if the distributions $f\left(x_{0}^{*}\right)$ and $f\left(x_{1}^{*}\right)$ can be efficiently distinguished with advantage at most $\Delta=\Delta(\lambda)$ to begin with (where $\Delta$ does not necessarily have to be negligible), then we require that no adversary that

\footnotetext{
${ }^{7}$ In personal communication with the authors of $\left[\mathrm{GJK}^{+} 13\right]$ we have learned that their work has been revised to take into account various definitional issues. The following comparison is based on the ePrint version of their work (as of October 7, 2014), and on this personal communication, and represents our current understanding of their revised notions of message privacy.
} 
is given a functional key for $f$ will be able to distinguish between encryptions of $x_{0}^{*}$ and $x_{1}^{*}$ with advantage larger than $\Delta+\operatorname{neg}(\lambda)$, for some negligible function neg $(\cdot)$.

This seems to better capture the motivating applications given by Goyal et al. of auditing an encrypted database and of performing differentially-private analysis on an encrypted database. In both applications the distributions obtained from two different encrypted databases are not negligibly close, as otherwise no utility can be gained.

- Goyal et al. allow adversaries to obtain functional keys only for functions $f$ for which the distributions $f\left(x_{0}^{*}\right)$ and $f\left(x_{1}^{*}\right)$ are negligibly close even when conditioned on the adversary's randomness and on the master secret key of the scheme. This may cause a situation where it is legal to obtain a functional key for some function $f$ by some specific adversary and for some specific instantiation of the functional encryption scheme, but it may be illegal to obtain a functional key for the same function $f$ by some other adversary and for some other instantiation of the encryption scheme.

In our framework, on one hand, each function family is considered either admissible or inadmissible independently of the adversary (and of the challenge ciphertext) and of the encryption scheme under consideration. However, on the other hand, our framework may consider some function families inadmissible, whereas the framework of Goyal et al. would consider the same function families as admissible with respect to some adversaries and encryption schemes. Nevertheless, our framework is still sufficiently general for capturing applications such as auditing an encrypted database, performing differentially-private analysis on an encrypted database, and many more.

\section{B Proofs of Claims $4.3-4.7$}

Proof of Claim 4.3. The adversary $\mathcal{B}^{(0) \rightarrow(1)}=\left(\mathcal{B}_{1}, \mathcal{B}_{2}\right)$ is defined as follows. First, $\mathcal{B}_{1}$ samples $T$ PRF keys $K_{1}, \ldots, K_{T} \leftarrow$ PRF.Gen $\left(1^{\lambda}\right)$ and $T$ functions $f_{1}, \ldots, f_{T} \leftarrow \mathcal{F}$ independently and uniformly at random. Then, it outputs the functions Left $_{f_{1}, K_{1}}, \ldots$, Left $_{f_{T}, K_{T}} \in \mathcal{F}^{\prime}$ (and its own randomness as the state information state).

Next, $\mathcal{B}_{2}$ on input functional keys $\mathrm{sk}_{1}, \ldots, \mathrm{sk}_{T}$ emulates the execution of $\mathcal{A}_{1}$ on input $\left(f_{1}, \ldots, f_{T}\right.$, $\left.\mathrm{sk}_{1}, \ldots, \mathrm{sk}_{T}\right)$ by simulating the encryption oracle $\operatorname{Enc}(\mathrm{msk}, \cdot)$ as follows: Whenever $\mathcal{A}_{1}$ requests an encryption of some $x \in \mathcal{X}_{\lambda}, \mathcal{B}_{2}$ samples $s \in\{0,1\}^{\lambda}$ uniformly at random, queries the encryption oracle FE.Enc $\sigma_{\sigma}(\mathrm{msk}, \cdot, \cdot)$ with the pair $((x, \perp, s, \perp),(x, x, s, \perp))$, and returns the answer to $\mathcal{A}_{1}$. When $\mathcal{A}_{1}$ outputs its challenge messages $\left(x_{0}^{*}, x_{1}^{*}\right), \mathcal{B}_{2}$ chooses a random bit $b$, samples $s^{*} \in\{0,1\}^{\lambda}$, and queries the encryption oracle $\mathrm{Enc}_{\sigma}(\mathrm{msk}, \cdot, \cdot)$ with the pair $\left(\left(x_{b}^{*}, \perp, s^{*}, \perp\right),\left(x_{b}^{*}, x_{b}^{*}, s^{*}, \perp\right)\right)$ to get the ciphertext $c^{*}$. Then, $\mathcal{B}_{2}$ runs $\mathcal{A}_{2}$ similarly to running $\mathcal{A}_{1}$ using the input $\left(c^{*}\right.$, state) to get its output $b^{\prime}$. Finally, $\mathcal{B}_{2}$ outputs 1 if $b^{\prime}=b$, and otherwise it outputs 0 .

Note that when $\sigma=0$ (the mode of the encryption oracle $\operatorname{Enc}_{\sigma}(\mathrm{msk}, \cdot, \cdot)$ ) then $\mathcal{A}$ 's view is identical to its view in the experiment $\mathcal{H}^{(0)}$, and when $\sigma=1$ then $\mathcal{A}$ 's view is identical to its view in the experiment $\mathcal{H}^{(1)}$. Therefore,

$$
\operatorname{Adv}_{\mathrm{FE}, \mathcal{F}^{\prime}, \mathcal{B}^{(0) \rightarrow(1)}, T}^{\mathrm{sfMP}}(\lambda)=\left|\operatorname{Pr}\left[\mathcal{H}^{(0)}(\lambda)=1\right]-\operatorname{Pr}\left[\mathcal{H}^{(1)}(\lambda)=1\right]\right| .
$$

Proof of Claim 4.4. The adversary $\mathcal{B}^{(2, i) \rightarrow(3, i)}=\left(\mathcal{B}_{1}, \mathcal{B}_{2}\right)$ is defined as follows. First, $\mathcal{B}_{1}$ chooses $T$ PRF keys $K_{1}, \ldots, K_{T} \leftarrow$ PRF.Gen $\left(1^{\lambda}\right)$ and $T$ functions $f_{1}, \ldots, f_{T} \leftarrow \mathcal{F}$ independently and uniformly at random. Then, it outputs the functions $\left(\right.$ Right $_{f_{1}, K_{1}}, \ldots$, Right $_{f_{i-1}, K_{i-1}}$, Left $f_{i}, K_{i}, \ldots$, Left $_{f_{T}, K_{T}}$ ) (and its own randomness as the state information state). 
Next, $\mathcal{B}_{2}$ on input functional keys $\mathrm{sk}_{1}, \ldots, \mathrm{sk}_{T}$ emulates the execution of $\mathcal{A}_{1}$ on input $\left(f_{1}, \ldots, f_{T}\right.$, $\left.\mathrm{sk}_{1}, \ldots, \mathrm{sk}_{T}\right)$ by simulating the encryption oracle Enc(msk, $)$ as follows: When $\mathcal{A}_{1}$ requests an encryption of $x \in \mathcal{X}_{\lambda}, \mathcal{B}_{2}$ samples $s \in\{0,1\}^{\lambda}$ uniformly at random, queries the encryption oracle $\operatorname{Enc}_{\sigma}(\mathrm{msk}, \cdot, \cdot)$ with the pair $\left((x, x, s, \perp),\left(x, x, s, f_{i}\left(x ; \operatorname{PRF} . \operatorname{Eval}\left(K_{i}, s\right)\right)\right)\right)$, and returns the answer to $\mathcal{A}_{1}$. When $\mathcal{A}_{1}$ outputs its challenge messages $\left(x_{0}^{*}, x_{1}^{*}\right), \mathcal{B}_{2}$ chooses a random bit $b$, samples $s^{*} \in\{0,1\}^{\lambda}$, and queries the encryption oracle Enc $\sigma(\operatorname{msk}, \cdot, \cdot)$ with the pair $\left(\left(x_{b}^{*}, x_{1}^{*}, s^{*}, \perp\right)\right.$, $\left(x_{b}^{*}, x_{1}^{*}, s^{*}, f_{i}\left(x_{b}^{*} ;\right.\right.$ PRF.Eval $\left.\left.\left.\left(K_{i}, s^{*}\right)\right)\right)\right)$ to get the ciphertext $c^{*}$. Then, $\mathcal{B}_{2}$ runs $\mathcal{A}_{2}$ similarly to running $\mathcal{A}_{1}$ using the input $\left(c^{*}\right.$, state) to get its output $b^{\prime}$. Finally, $\mathcal{B}_{2}$ outputs 1 if $b^{\prime}=b$, and otherwise it outputs 0 .

Note that when $\sigma=0$ (the mode of the encryption oracle Enc $\sigma(\mathrm{msk}, \cdot, \cdot)$ ) then $\mathcal{A}$ 's view is identical to its view in the experiment $\mathcal{H}^{(2, i)}$, and when $\sigma=1$ the $\mathcal{A}$ 's view is identical to its view in the experiment $\mathcal{H}^{(3, i)}$. Therefore,

$$
\operatorname{Adv}_{\mathrm{FE}, \mathcal{F}^{\prime}, \mathcal{B}^{(2, i)} \rightarrow(3, i), T}^{\mathrm{sfMP}}(\lambda)=\left|\operatorname{Pr}\left[\mathcal{H}^{(2, i)}(\lambda)=1\right]-\operatorname{Pr}\left[\mathcal{H}^{(3, i)}(\lambda)=1\right]\right| .
$$

Proof of Claim 4.5. The adversary $\mathcal{B}^{(3, i) \rightarrow(4, i)}=\left(\mathcal{B}_{1}, \mathcal{B}_{2}\right)$ is defined as follows. First, $\mathcal{B}_{1}$ chooses $T$ PRF keys $K_{1}, \ldots, K_{T} \leftarrow$ PRF.Gen $\left(1^{\lambda}\right)$ and $T$ functions $f_{1}, \ldots, f_{T} \leftarrow \mathcal{F}$ independently and uniformly at random. Then, it outputs the functions $\left(\operatorname{Right}_{f_{1}, K_{1}}, \ldots\right.$, Right $_{f_{i-1}, K_{i-1}}$, Left $_{f_{i}, K_{i}}, \ldots$, Left $_{f_{T}, K_{T}}$ ) and (Right $_{f_{1}, K_{1}}, \ldots$, Right $_{f_{i-1}, K_{i-1}}$, OutputZ, Left $f_{i+1}, K_{i+1}, \ldots$, Left $f_{T}, K_{T}$ ) (and its own randomness as the state information state).

Next, $\mathcal{B}_{2}$ on input functional keys $\mathrm{sk}_{1}, \ldots, \mathrm{sk}_{T}$ emulates the execution of $\mathcal{A}_{1}$ on input $\left(f_{1}, \ldots, f_{T}\right.$, $\left.\mathrm{sk}_{1}, \ldots, \mathrm{sk}_{T}\right)$ by simulating the encryption oracle Enc(msk, $\left.\cdot\right)$ as follows: When $\mathcal{A}_{1}$ requests an encryption of $x \in \mathcal{X}_{\lambda}, \mathcal{B}_{2}$ samples $s \in\{0,1\}^{\lambda}$ uniformly at random, queries the encryption oracle Enc $_{\sigma}(\mathrm{msk}, \cdot, \cdot)$ with the pair $\left(\left(x, x, s, f_{i}\left(x ; \operatorname{PRF} . \operatorname{Eval}\left(K_{i}, s\right)\right)\right),\left(x, x, s, f_{i}\left(x ; \operatorname{PRF} . \operatorname{Eval}\left(K_{i}, s\right)\right)\right)\right)$, and returns the answer to $\mathcal{A}_{1}$. When $\mathcal{A}_{1}$ outputs its challenge messages $\left(x_{0}^{*}, x_{1}^{*}\right), \mathcal{B}_{2}$ chooses a ran-

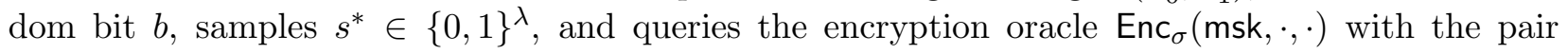
$\left(\left(x_{b}^{*}, x_{1}^{*}, s^{*}, f_{i}\left(x_{b}^{*}\right.\right.\right.$; PRF.Eval $\left.\left.\left(K_{i}, s^{*}\right)\right)\right),\left(x_{b}^{*}, x_{1}^{*}, s^{*}, f_{i}\left(x_{b}^{*}\right.\right.$; PRF.Eval $\left.\left.\left.\left(K_{i}, s^{*}\right)\right)\right)\right)$ to get the ciphertext $c^{*}$. Then, $\mathcal{B}_{2}$ runs $\mathcal{A}_{2}$ similarly to running $\mathcal{A}_{1}$ using the input $\left(c^{*}\right.$, state) to get its output $b^{\prime}$. Finally, $\mathcal{B}_{2}$ outputs 1 if $b^{\prime}=b$, and otherwise it outputs 0 .

Note that when $\sigma=0$ (the functional keys correspond to the first list of functions) then $\mathcal{A}$ 's view is identical to its view in the experiment $\mathcal{H}^{(3, i)}$, and when $\sigma=1$ then $\mathcal{A}$ 's view is identical to its view in the experiment $\mathcal{H}^{(4, i)}$. Therefore,

$$
\operatorname{Adv}_{\mathrm{FE}, \mathcal{F}^{\prime}, \mathcal{B}^{(3, i) \rightarrow(4, i)}, T}^{\mathrm{sfPP}}(\lambda)=\left|\operatorname{Pr}\left[\mathcal{H}^{(3, i)}(\lambda)=1\right]-\operatorname{Pr}\left[\mathcal{H}^{(4, i)}(\lambda)=1\right]\right| .
$$

Proof of Claim 4.6. The adversary $\mathcal{B}^{(4, i) \rightarrow(5, i)}=\mathcal{B}$ is defined as follows. First, $\mathcal{B}$ chooses a master key msk $\leftarrow$ FE.Setup $\left(1^{\lambda}\right), T-1$ PRF keys $K_{1}, \ldots, K_{i-1}, K_{i+1}, \ldots, K_{T} \leftarrow$ PRF.Gen $\left(1^{\lambda}\right)$ and $T$ functions $f_{1}, \ldots, f_{T} \leftarrow \mathcal{F}$ independently and uniformly at random. Then, it computes the functional keys $\mathrm{sk}_{1}, \ldots, \mathrm{sk}_{T}$ for the functions (Right $f_{f_{1}, K_{1}}, \ldots$, Right $_{f_{i-1}, K_{i-1}}$, OutputZ, Left $f_{i+1}, K_{i+1}, \ldots$, Left $_{f_{T}, K_{T}}$ ) using msk. Recall that $\mathcal{B}$ has access to an oracle, denoted $R(\cdot)$, that is either a random function or a $\mathrm{PRF}$ and its goal is to distinguish between the two cases.

Next, $\mathcal{B}$ emulates the execution of $\mathcal{A}_{1}$ on input $\left(f_{1}, \ldots, f_{T}, \mathrm{sk}_{1}, \ldots, \mathrm{sk}_{T}\right)$ by simulating the encryption oracle Enc $(\mathrm{msk}, \cdot)$ as follows: When $\mathcal{A}_{1}$ requests an encryption of $x \in \mathcal{X}_{\lambda}$, $\mathcal{B}$ samples $s \in\{0,1\}^{\lambda}$ uniformly at random, queries $R(s)$ to get $r$, computes FE.Enc(msk, $\left.\left(x, x, s, f_{i}(x ; r)\right)\right)$, and returns the output to $\mathcal{A}_{1}$. When $\mathcal{A}_{1}$ outputs its challenge messages $\left(x_{0}^{*}, x_{1}^{*}\right), \mathcal{B}$ chooses a 
random bit $b$, samples $s^{*} \in\{0,1\}^{\lambda}$, queries $R\left(s^{*}\right)$ to get $r^{*}$ and computes the ciphertext $c^{*}=$ FE.Enc $\left(\mathrm{msk},\left(x_{b}^{*}, x_{1}^{*}, s^{*}, f_{i}\left(x_{b}^{*} ; r^{*}\right)\right)\right)$. Then, $\mathcal{B}$ runs $\mathcal{A}_{2}$ similarly to running $\mathcal{A}_{1}$ using the input $\left(c^{*}\right.$, state) to get its output $b^{\prime}$. Finally, $\mathcal{B}_{2}$ outputs 1 if $b^{\prime}=b$, and otherwise it outputs 0 .

Note that when $R(\cdot)$ corresponds to a pseudorandom function then $\mathcal{A}$ 's view is identical to its view in the experiment $\mathcal{H}^{(4, i)}$, and when $R(\cdot)$ corresponds to a truly random function then $\mathcal{A}$ 's view is identical to its view in the experiment $\mathcal{H}^{(5, i)}$. Therefore,

$$
\operatorname{Adv}_{\mathrm{PRF}, \mathcal{B}^{(4, i) \rightarrow(5, i)}}(\lambda)=\left|\operatorname{Pr}\left[\mathcal{H}^{(4, i)}(\lambda)=1\right]-\operatorname{Pr}\left[\mathcal{H}^{(5, i)}(\lambda)=1\right]\right| .
$$

Proof of Claim 4.7. The adversary $\mathcal{B}^{(5, i) \rightarrow(6, i)}=\left(\mathcal{B}_{1}, \mathcal{B}_{2}\right)$ on input $f \leftarrow \mathcal{F}$ is defined as follows. First, $\mathcal{B}_{1}$ chooses a master key msk $\leftarrow$ FE.Setup $\left(1^{\lambda}\right), T$ PRF keys $K_{1}, \ldots, K_{T} \leftarrow$ PRF.Gen $\left(1^{\lambda}\right)$ and $T-1$ functions $f_{1}, \ldots, f_{i-1}, f_{i+1}, \ldots, f_{T} \leftarrow \mathcal{F}$ independently and uniformly at random. Then, it computes the functional keys $\mathrm{sk}_{1}, \ldots, \mathrm{sk}_{T}$ for the functions $\left(\operatorname{Right}_{f_{1}, K_{1}}, \ldots\right.$, $\operatorname{Right}_{f_{i-1}, K_{i-1}}$, OutputZ, Left $_{f_{i+1}, K_{i+1}}, \ldots$, Left $\left._{f_{T}, K_{T}}\right)$. Next, $\mathcal{B}_{1}$ emulates the execution of $\mathcal{A}_{1}$ on input $\left(f_{1}, \ldots, f_{i-1}, f, f_{i+1}\right.$, $\left.\ldots, f_{T}, \mathrm{sk}_{1}, \ldots, \mathrm{sk}_{T}\right)$ by simulating the encryption oracle Enc(msk, $\left.\cdot\right)$ as follows: When $\mathcal{A}_{1}$ requests an encryption of $x \in \mathcal{X}_{\lambda}, \mathcal{B}_{2}$ samples $s \in\{0,1\}^{\lambda}$ and a random string $r$, computes FE.Enc(msk, $(x, x, s, f(x ; r)))$, and returns the output to $\mathcal{A}_{1}$. When $\mathcal{A}_{1}$ outputs its challenge messages $\left(x_{0}^{*}, x_{1}^{*}\right)$, $\mathcal{B}_{2}$ outputs $\left(x_{b}^{*}, x_{1}^{*}\right)$, where $b \in\{0,1\}$ is chosen uniformly at random.

Then, $\mathcal{B}_{2}$ on input $y$ (which is a uniform sample either from $f\left(x_{b}^{*}\right)$ or from $f\left(x_{1}^{*}\right)$ ) samples $s^{*} \in\{0,1\}^{\lambda}$ uniformly at random, computes $c^{*}=$ FE.Enc $\left(m s k,\left(x_{b}^{*}, x_{1}^{*}, s^{*}, y\right)\right)$, runs $\mathcal{A}_{2}$ similarly to running $\mathcal{A}_{1}$ using the input $c^{*}$ to get its output $b^{\prime}$. Finally, $\mathcal{B}_{2}$ outputs 1 if $b^{\prime}=b$, and otherwise it outputs 0 .

Note that when $y$ is sampled according to $f\left(x_{b}^{*}\right)$, then $\mathcal{A}$ 's view is identical to its view in the experiment $\mathcal{H}^{(5, i)}$. Similarly, when $y$ is sampled according to $f\left(x_{1}^{*}\right)$, then $\mathcal{A}$ 's view is identical to its view in the experiment $\mathcal{H}^{(6, i)}$. Therefore,

$$
\operatorname{Adv}_{\mathcal{F}, \mathcal{B}^{(5, i) \rightarrow(6, i)}}^{\mathrm{naADM}}(\lambda)=\left|\operatorname{Pr}\left[\mathcal{H}^{(5, i)}(\lambda)=1\right]-\operatorname{Pr}\left[\mathcal{H}^{(6, i)}(\lambda)=1\right]\right|
$$

\section{Proofs of Claims 4.12-4.19}

Proof of Claim 4.12. The adversary $\mathcal{B}^{(0) \rightarrow(1)}=\mathcal{B}$ is defined as follows. First, $\mathcal{B}$ emulates the execution of $\mathcal{A}_{1}$ on input $\left(1^{\lambda}\right)$ by simulating the encryption oracle and the key generation oracle as follows: When $\mathcal{A}_{1}$ requests an encryption of $x \in \mathcal{X}_{\lambda}, \mathcal{B}$ samples $s \in\{0,1\}^{\lambda}$, queries the encryption oracle Enc $_{\sigma}(\mathrm{msk}, \cdot, \cdot)$ with the pair $((x, \perp, s, \perp),(x, \perp, s, \perp))$ and returns the output to $\mathcal{A}_{1}$. When $\mathcal{A}_{1}$ requests a functional key for $f \in \mathcal{F}, \mathcal{B}$, samples a PRF key $K_{1} \leftarrow \operatorname{PRF}$.Gen $\left(1^{\lambda}\right)$, queries the key generation oracle $\mathrm{KG}\left(\mathrm{msk}\right.$, Left $\left.{ }_{f, K}\right)$ and returns the output to $\mathcal{A}_{1}$. Finally, $\mathcal{A}_{1}$ outputs the challenge $\left(x_{0}^{*}, x_{1}^{*}\right.$, state).

Next, $\mathcal{B}$ chooses a random bit $b$, samples $s^{*} \in\{0,1\}^{\lambda}$, and queries the encryption oracle with the pair $\left(\left(x_{b}^{*}, \perp, s^{*}, \perp\right),\left(x_{b}^{*}, x_{b}^{*}, s^{*}, \perp\right)\right)$ to get the ciphertext $c^{*}$. Then, $\mathcal{B}$ runs $\mathcal{A}_{2}$ similarly to running $\mathcal{A}_{1}$ using the input $\left(c^{*}\right.$, state) to get its output $b^{\prime}$. Finally, $\mathcal{B}$ outputs 1 if $b^{\prime}=b$, and otherwise it outputs 0 .

Note that when $\sigma=0$ (the mode of the encryption oracle $\operatorname{Enc}_{\sigma}(\mathrm{msk}, \cdot, \cdot)$ ) then $\mathcal{A}$ 's view is identical to its view in the experiment $\mathcal{H}^{(0)}$, and when $\sigma=1$ then $\mathcal{A}^{\prime}$ 's view is identical to its view in the experiment $\mathcal{H}^{(1)}$. Therefore,

$$
\left|\operatorname{Pr}\left[\mathcal{H}^{(0)}(\lambda)=1\right]-\operatorname{Pr}\left[\mathcal{H}^{(1)}(\lambda)=1\right]\right|=\operatorname{Adv}_{\mathrm{FE}, \mathcal{F}^{\prime}, \mathcal{B}}^{\mathrm{MP}(0) \rightarrow(1), T}(\lambda) .
$$


Proof of Claim 4.13. The adversary $\mathcal{B}^{(1) \rightarrow(2,1)}=\mathcal{B}$ is defined as follows. First, $\mathcal{B}$ emulates the execution of $\mathcal{A}_{1}$ on input $\left(1^{\lambda}\right)$ by simulating the encryption oracle and the key generation oracle as follows: When $\mathcal{A}_{1}$ requests an encryption of $x \in \mathcal{X}_{\lambda}, \mathcal{B}$ samples $s \in\{0,1\}^{\lambda}$, queries the encryption oracle $\mathrm{Enc}_{\sigma}(\mathrm{msk}, \cdot, \cdot)$ with the pair $((x, \perp, s, \perp),(x, \perp, s, \perp))$ and returns the output to $\mathcal{A}_{1}$. When $\mathcal{A}_{1}$ requests a functional key for $f \in \mathcal{F}, \mathcal{B}$ samples a PRF key $K \leftarrow \operatorname{PRF} . G e n\left(1^{\lambda}\right)$, queries the key generation oracle $\mathrm{KG}_{\sigma}(\mathrm{msk}, \cdot, \cdot)$ with the pair $\left(\operatorname{Left}_{f, K}\right.$, Left $\left.f, K\right)$ and returns the output to $\mathcal{A}_{1}$. Finally, $\mathcal{A}_{1}$ outputs the challenge $\left(x_{0}^{*}, x_{1}^{*}\right.$, state).

Next, $\mathcal{B}$ chooses a random bit $b$, samples $s^{*} \in\{0,1\}^{\lambda}$, and queries the encryption oracle with the pair $\left(\left(x_{b}^{*}, \perp, s^{*}, \perp\right),\left(x_{b}^{*}, x_{b}^{*}, s^{*}, \perp\right)\right)$ to get the ciphertext $c^{*}$. We assume that $s^{*} \neq s$ for all sampled $s^{\prime}$ 's and lose an additive negligible factor. Then, $\mathcal{B}$ emulates the execution of $\mathcal{A}_{2}$ on input ( $c^{*}$, state) by simulation the encryption oracle in the same way and simulating the key generation oracle as follows: When $\mathcal{A}_{2}$ requests for a functional key for $f \in \mathcal{F}, \mathcal{B}$ samples $K \leftarrow$ PRF.Gen $\left(1^{\lambda}\right)$ computes the key $K^{\prime}$ by puncturing $K$ at $s^{*}$, sets $y=f\left(x_{b}^{*}\right.$; PRF.Eval $\left.K_{K}\left(s^{*}\right)\right)$ and queries the key generation oracle $\mathrm{KG}_{\sigma}(\mathrm{msk}, \cdot, \cdot)$ with the pair ( Left $_{f, K}$, PuncOutput $\left._{f, K^{\prime}, y, s^{*}}\right)$. Finally, when $\mathcal{A}_{2}$ outputs $b^{\prime}, \mathcal{B}$ outputs 1 if $b^{\prime}=b$, and otherwise it outputs 0 .

Note that when $\sigma=0$ (the mode of the encryption oracle Enc E $_{\sigma}(\mathrm{msk}, \cdot, \cdot)$ ) and key generation oracle $\left.\mathrm{KG}_{\sigma}(\mathrm{msk}, \cdot, \cdot)\right)$ then $\mathcal{A}$ 's view is identical to its view in the experiment $\mathcal{H}^{(1)}$, and when $\sigma=1$ then $\mathcal{A}$ 's view is identical to its view in the experiment $\mathcal{H}^{(2,1)}$. Therefore,

$$
\left|\operatorname{Pr}\left[\mathcal{H}^{(1)}(\lambda)=1\right]-\operatorname{Pr}\left[\mathcal{H}^{(2,1)}(\lambda)=1\right]\right| \leq \operatorname{Adv}_{\mathrm{FE}, \mathcal{F}^{\prime}, \mathcal{B}^{(1)} \rightarrow(2,1)}^{\mathrm{FP}}(\lambda)+\operatorname{neg}(\lambda) .
$$

Proof of Claim 4.14. The adversary $\mathcal{B}^{(2, i) \rightarrow(2, i+1)}=\left(\mathcal{B}_{1}, \mathcal{B}_{2}\right)$ is defined as follows. $\mathcal{B}_{1}$ samples a random string $s^{*} \in\{0,1\}^{\lambda}$ and outputs the set $\left\{s^{*}\right\}$ as the punctured set and the point $s^{*}$ as the state.

$\mathcal{B}_{2}$ gets as input a punctured PRF key $K^{\prime} \triangleq K_{\left\{s^{*}\right\}}$ at the point $s^{*}$, a value $y$ (which is either $\operatorname{PRF}_{K}\left(s^{*}\right)$ or a random string), and the point $s^{*}$ as the state information. Next, $\mathcal{B}_{2}$ chooses a master key msk $\leftarrow$ FE.Setup $\left(1^{\lambda}\right)$ and emulates the execution of $\mathcal{A}_{1}$ on input $1^{\lambda}$ by simulating the encryption oracle and the key generation oracle as follows: When $\mathcal{A}_{1}$ requests an encryption of $x \in \mathcal{X}_{\lambda}, \mathcal{B}_{2}$ samples $s \in\{0,1\}^{\lambda}$, computes FE.Enc (msk, $\left.(x, \perp, s, \perp)\right)$ and returns the output to $\mathcal{A}_{1}$. When $\mathcal{A}_{1}$ requests a functional key for the function $f \in \mathcal{F}, \mathcal{B}_{2}$ samples a PRF key $K \leftarrow$ PRF.Gen $\left(1^{\lambda}\right)$, computes FE.KG $($ msk, Left $f, K)$, and returns the output to $\mathcal{A}_{1}$. Finally, $\mathcal{A}_{1}$ outputs the challenge $\left(x_{0}^{*}, x_{1}^{*}\right.$, state).

Then, $\mathcal{B}_{2}$ chooses a random bit $b$, computes $c^{*} \leftarrow$ FE.Enc $\left(\operatorname{msk},\left(x_{b}^{*}, x_{1}^{*}, s^{*}, \perp\right)\right)$ and emulates the execution of $\mathcal{A}_{2}$ on input $\left(c^{*}\right.$, state) by simulating the encryption oracle as before and simulating the key generation oracle as follows: When $\mathcal{A}_{2}$ requests the $j^{\text {th }}$ functional key for $f_{j} \in \mathcal{F}$, if $j<i$ then $\mathcal{B}_{2}$ samples a PRF key $K_{j} \leftarrow$ PRF.Gen $\left(1^{\lambda}\right)$, obtains $K_{j}^{\prime}$ by puncturing $K_{j}$ at $s^{*}$, samples $y \leftarrow f\left(x_{b}^{*}\right)$, computes FE.KG(msk, PuncOutputY $\left.{ }_{f_{j}, K_{j}^{\prime}, y, s^{*}}\right)$ and returns the output to $\mathcal{A}_{2}$. If $j=i$ then $\mathcal{B}_{2}$, computes FE.KG(msk, PuncOutputY $\left.{ }_{f_{j}, K^{\prime}, y, s^{*}}\right)$ and returns the output to $\mathcal{A}_{2}$. If $j>i$ then $\mathcal{B}_{2}$ samples a PRF key $K_{j} \leftarrow$ PRF.Gen $\left(1^{\lambda}\right)$, obtains $K_{j}^{\prime}$ by puncturing $K_{j}$ at $s^{*}$, samples $y=f\left(x_{b}^{*}\right.$; PRF.Eval $\left.K_{j}\left(s^{*}\right)\right)$, computes FE.KG(msk, PuncOutputY $\left._{f_{j}, K_{j}^{\prime}, y, s^{*}}\right)$ and returns the output to $\mathcal{A}_{2}$. Finally, when $\mathcal{A}_{2}$ outputs the bit $b^{\prime}, \mathcal{B}_{2}$ outputs 1 if $b^{\prime}=b$, and otherwise it outputs 0 .

Note that when $y$ is obtained from as a PRF evaluation then $\mathcal{A}$ 's view is identical to its view in the experiment $\mathcal{H}^{(2, i+1)}$, and when $y$ is obtained from as a random string then $\mathcal{A}$ 's view is identical to its view in the experiment $\mathcal{H}^{(2, i)}$. Therefore,

$$
\left|\operatorname{Pr}\left[\mathcal{H}^{(2, i)}(\lambda)=1\right]-\operatorname{Pr}\left[\mathcal{H}^{(2, i+1)}(\lambda)=1\right]\right|=\operatorname{Adv}_{\text {puPRF }, \mathcal{B}^{(2, i) \rightarrow(2, i+1)}}(\lambda) .
$$


Proof of Claim 4.15. The adversary $\mathcal{B}^{(3, i) \rightarrow(3, i+1)}=\left(\mathcal{B}_{1}, \mathcal{B}_{2}\right)$ is defined as follows. First, $\mathcal{B}_{1}$ chooses a master key msk $\leftarrow$ FE.Setup $\left(1^{\lambda}\right)$ and emulates the execution of $\mathcal{A}_{1}$ on input $1^{\lambda}$ by simulating the encryption oracle and the key generation oracle as follows: When $\mathcal{A}_{1}$ requests an encryption of $x \in \mathcal{X}_{\lambda}, \mathcal{B}_{1}$ samples $s \in\{0,1\}^{\lambda}$, computes FE.Enc(msk, $\left.(x, \perp, s, \perp)\right)$ and returns the output to $\mathcal{A}_{1}$. When $\mathcal{A}_{1}$ requests a functional key for the function $f \in \mathcal{F}, \mathcal{B}_{1}$ samples a PRF key $K \leftarrow$ PRF.Gen $\left(1^{\lambda}\right)$, computes FE.KG $\left(\right.$ msk, Left $\left._{f, K}\right)$, and returns the output to $\mathcal{A}_{1}$. When, $\mathcal{A}_{1}$ outputs the challenge $\left(x_{0}^{*}, x_{1}^{*}\right.$, state $), \mathcal{B}_{1}$ chooses a random bit $b$, computes $c^{*} \leftarrow$ FE.Enc $\left(\operatorname{msk},\left(x_{b}^{*}, x_{1}^{*}, s^{*}, \perp\right)\right)$ and emulates the execution of $\mathcal{A}_{2}$ on input ( $c^{*}$, state) by simulating the encryption oracle as before and simulating the key generation oracle for the first $i-1$ times as follows: When $\mathcal{A}_{2}$ requests a functional key for $f \in \mathcal{F}, \mathcal{B}_{1}$ samples a PRF key $K \leftarrow$ PRF.Gen $\left(1^{\lambda}\right)$, obtains $K^{\prime}$ by puncturing $K$ at $s^{*}$, samples $y \leftarrow f\left(x_{1}^{*}\right)$, computes FE.KG(msk, PuncOutputY $\left.{ }_{f, K^{\prime}, y, s^{*}}\right)$ and returns the output to $\mathcal{A}_{2}$. When $\mathcal{A}_{2}$ requests the $i^{\text {th }}$ functional key $f \in \mathcal{F}$ then $\mathcal{B}_{1}$ chooses a random bit $b$, outputs $\left(f, x_{b}^{*}, x_{1}^{*}\right)$ and its entire memory and internal randomness as the state state (without answering the last key generation query of $\mathcal{A}_{2}$ ).

Next, $\mathcal{B}_{2}$ runs on input ( $y$, state), continues the execution of $\mathcal{A}_{2}$, samples a PRF key $K \leftarrow$ PRF.Gen $\left(1^{\lambda}\right)$, obtains $K^{\prime}$ by puncturing $K$ at $s^{*}$, computes FE.KG(msk, PuncOutput $\left.Y_{f, K^{\prime}, y, s^{*}}\right)$ and returns the output to $\mathcal{A}_{2}$. When $\mathcal{A}_{2}$ requests a functional key for the function $f \in \mathcal{F}, \mathcal{B}_{2}$ samples a PRF key $K \leftarrow$ PRF.Gen $\left(1^{\lambda}\right)$, obtains $K^{\prime}$ by puncturing $K$ at $s^{*}$, samples $y=f\left(x_{b}^{*}\right.$; PRF.Eval $\left.K\left(s^{*}\right)\right)$, computes FE.KG(msk, PuncOutputY $\left.Y_{f, K^{\prime}, y, s^{*}}\right)$ and returns the output to $\mathcal{A}_{2}$. Finally, when $\mathcal{A}_{2}$ outputs the bit $b^{\prime}, \mathcal{B}_{2}$ outputs 1 if $b^{\prime}=b$, and otherwise it outputs 0 .

Note that when $y$ is sampled from $f\left(x_{b}^{*}\right)$ then $\mathcal{A}$ 's view is identical to its view in the experiment $\mathcal{H}^{(3, i+1)}$, and when $y$ is sampled from $f\left(x_{1}^{*}\right)$ then $\mathcal{A}$ 's view is identical to its view in the experiment $\mathcal{H}^{(3, i)}$. Therefore,

$$
\left|\operatorname{Pr}\left[\mathcal{H}^{(3, i)}(\lambda)=1\right]-\operatorname{Pr}\left[\mathcal{H}^{(3, i+1)}(\lambda)=1\right]\right|=\operatorname{Adv}_{\mathcal{F}, \mathcal{B}^{(3, i) \rightarrow(3, i+1)}}^{\mathrm{aADM}} \leq \Delta(\lambda) .
$$

Proof of Claim 4.16. The adversary $\mathcal{B}^{(4, i) \rightarrow(5, i)}=\mathcal{B}$ is defined as follows. First, $\mathcal{B}$ samples $T_{1}$ PRF keys $K_{1}, \ldots, K_{T_{1}} \leftarrow$ PRF.Gen $\left(1^{\lambda}\right)$. Then, $\mathcal{B}$ emulates the execution of $\mathcal{A}_{1}$ on input $\left(1^{\lambda}\right)$ by simulating the encryption oracle and the key generation oracle as follows: When $\mathcal{A}_{1}$ requests an encryption of $x \in \mathcal{X}_{\lambda}, \mathcal{B}$ samples $s \in\{0,1\}^{\lambda}$, queries the encryption oracle Enc $\sigma(\mathrm{msk}, \cdot, \cdot)$ with the pair $((x, \perp, s, \perp),(x, \perp, s, \perp))$ and returns the output to $\mathcal{A}_{1}$. When $\mathcal{A}_{1}$ requests a functional key for the function $f \in \mathcal{F}$ for the $j^{\text {th }}$ time, if $j \leq i-1$ then $\mathcal{B}$ queries the key generation oracle $\mathrm{KG}\left(\mathrm{msk}\right.$, Right $\left.{ }_{f, K_{j}}\right)$ and returns the output to $\mathcal{A}_{1}$, and otherwise $\mathcal{B}$ queries the key generation oracle with KG(msk, Left $\left.{ }_{f, K_{j}}\right)$ and returns the output to $\mathcal{A}_{1}$. Finally, $\mathcal{A}_{1}$ outputs the challenge $\left(x_{0}^{*}, x_{1}^{*}\right.$, state).

Next, $\mathcal{B}$ chooses a random bit $b$, samples $s^{*} \in\{0,1\}^{\lambda}$, computes $z^{*}=f_{i}\left(x_{b}^{*}\right.$; PRF.Eval $\left.K_{i}\left(s^{*}\right)\right)$ and queries the encryption oracle with the pair $\left(\left(x_{b}^{*}, x_{1}^{*}, s^{*}, \perp\right),\left(x_{b}^{*}, x_{1}^{*}, s^{*}, z^{*}\right)\right)$ to get the ciphertext $c^{*}$. Then, $\mathcal{B}$ emulates the execution of $\mathcal{A}_{2}$ on input $\left(c^{*}\right.$, state $)$ by simulating the key generation oracle as follows: When $\mathcal{A}_{2}$ requests a functional key for the function $f \in \mathcal{F}$, B samples $s^{*} \in\{0,1\}^{\lambda}$ uniformly at random, samples $K \leftarrow$ PRF.Gen $\left(1^{\lambda}\right)$, computes the key $K^{\prime}$ which is the key $K$ punctured at the point $s^{*}$, queries the key generation oracle $\mathrm{KG}_{\sigma}(\mathrm{msk}, \cdot, \cdot)$ with the pair (PuncOutputZ $Z_{f_{i}, K^{\prime}, s^{*}}$, PuncOutputZ $\mathrm{f}_{i}, K^{\prime}, s^{*}$ ) and returns the output to $\mathcal{A}_{1}$. Finally, when $\mathcal{A}_{2}$ outputs the bit $b^{\prime}, \mathcal{B}$ outputs 1 if $b^{\prime}=b$, and otherwise it outputs 0 .

Note that when $\sigma=0$ (the mode of the encryption oracle Enc $\sigma(\mathrm{msk}, \cdot, \cdot)$ ) then $\mathcal{A}$ 's view is identical to its view in the experiment $\mathcal{H}^{(4, i)}$, and when $\sigma=1$ then $\mathcal{A}$ 's view is identical to its view 
in the experiment $\mathcal{H}^{(5, i)}$. Therefore,

$$
\left|\operatorname{Pr}\left[\mathcal{H}^{(4, i)}(\lambda)=1\right]-\operatorname{Pr}\left[\mathcal{H}^{(5, i)}(\lambda)=1\right]\right|=\operatorname{Adv}_{\mathrm{FE}, \mathcal{F}^{\prime}, \mathcal{B}^{(4, i) \rightarrow(5, i)}, T}^{\mathrm{MP}}(\lambda) .
$$

Proof of Claim 4.17. The adversary $\mathcal{B}^{(5, i) \rightarrow(6, i)}=\mathcal{B}$ is defined as follows. First, $\mathcal{B}$ samples $T_{1}$ PRF keys $K_{1}, \ldots, K_{T_{1}} \leftarrow$ PRF.Gen $\left(1^{\lambda}\right)$. Then, $\mathcal{B}$ emulates the execution of $\mathcal{A}_{1}$ on input $\left(1^{\lambda}\right)$ by simulating the encryption oracle and the key generation oracle as follows: When $\mathcal{A}_{1}$ requests an encryption of $x \in \mathcal{X}_{\lambda}, \mathcal{B}$ samples $s \in\{0,1\}^{\lambda}$, queries the encryption oracle Enc $_{\sigma}(\mathrm{msk}, \cdot, \cdot)$ with the pair $((x, \perp, s, \perp),(x, \perp, s, \perp))$ and returns the output to $\mathcal{A}_{1}$. When $\mathcal{A}_{1}$ requests a functional key for the function $f \in \mathcal{F}$ for the $j^{\text {th }}$ time, if $j<i$ then $\mathcal{B}$ queries the key generation oracle $\mathrm{KG}_{\sigma}(\mathrm{msk}, \cdot, \cdot)$ with the pair $\left(\operatorname{Right}_{f, K_{j}}\right.$, Right $\left._{f, K_{j}}\right)$ and returns the output to $\mathcal{A}_{1}$. If $j=i$ then $\mathcal{B}$ samples $s^{*} \in\{0,1\}^{\lambda}$ uniformly at random, samples a PRF key $K$, obtains a punctured key $K^{\prime}$ which is the key $K$ punctured at the point $s^{*}$, queries the key generation oracle $\mathrm{KG}_{\sigma}(\mathrm{msk}, \cdot, \cdot)$ with the pair $\left(\right.$ Right $_{f, K_{i}}$, PuncOutputZ $\left._{f_{i}, K_{i}^{\prime}, s^{*}}\right)$ and returns the output to $\mathcal{A}_{1}$. If $j>i$ then $\mathcal{B}$ queries the key generation oracle $\mathrm{KG}_{\sigma}(\mathrm{msk}, \cdot, \cdot)$ with the pair $\left(\operatorname{Left}_{f, K_{j}}\right.$, Left $f_{, K_{j}}$ ) and returns the output to $\mathcal{A}_{1}$. Finally, $\mathcal{A}_{1}$ outputs the challenge $\left(x_{0}^{*}, x_{1}^{*}\right.$, state).

Next, $\mathcal{B}$ chooses a random bit $b$, samples $s^{*} \in\{0,1\}^{\lambda}$, computes $z^{*}=f_{i}\left(x_{b}^{*}\right.$; PRF.Eval $\left.K_{i}\left(s^{*}\right)\right)$ and queries the encryption oracle with the pair $\left(\left(x_{b}^{*}, x_{1}^{*}, s^{*}, z^{*}\right),\left(x_{b}^{*}, x_{1}^{*}, s^{*}, z^{*}\right)\right)$ to get the ciphertext $c^{*}$. We assume that $s^{*} \neq s$ for all sampled $s^{\prime}$ s and lose an additive negligible factor. Then, $\mathcal{B}$ emulates the execution of $\mathcal{A}_{2}$ on input ( $c^{*}$, state) by simulating the key generation oracle as follows: When $\mathcal{A}_{2}$ requests a functional key for the function $f \in \mathcal{F}, \mathcal{B}$ samples $s^{*} \in\{0,1\}^{\lambda}$ uniformly at random, samples $K \leftarrow$ PRF.Gen $\left(1^{\lambda}\right)$, computes the key $K^{\prime}$ which is the key $K$ punctured at the point $s^{*}$, queries the key generation oracle $\mathrm{KG}_{\sigma}(\mathrm{msk}, \cdot, \cdot)$ with the pair (PuncOutputZ $Z_{f_{i}, K^{\prime}, s^{*}}$, PuncOutputZ $Z_{f_{i}, K^{\prime}, s^{*}}$ ) and returns the output to $\mathcal{A}_{1}$. Finally, when $\mathcal{A}_{2}$ outputs the bit $b^{\prime}, \mathcal{B}$ outputs 1 if $b^{\prime}=b$, and otherwise it outputs 0 .

Note that when $\sigma=0$ (the mode of the encryption oracle $\operatorname{Enc}_{\sigma}(\mathrm{msk}, \cdot, \cdot)$ ) then $\mathcal{A}$ 's view is identical to its view in the experiment $\mathcal{H}^{(5, i)}$, and when $\sigma=1$ then $\mathcal{A}$ 's view is identical to its view in the experiment $\mathcal{H}^{(6, i)}$. Therefore,

$$
\left|\operatorname{Pr}\left[\mathcal{H}^{(5, i)}(\lambda)=1\right]-\operatorname{Pr}\left[\mathcal{H}^{(6, i)}(\lambda)=1\right]\right| \leq \operatorname{Adv}_{\mathrm{FE}, \mathcal{F}^{\prime}, \mathcal{B}^{(5, i) \rightarrow(6, i)}, T}^{\mathrm{FP}}(\lambda)+\operatorname{neg}(\lambda) .
$$

Proof of Claim 4.18. The adversary $\mathcal{B}^{(6, i) \rightarrow(7, i)}=\left(\mathcal{B}_{1}, \mathcal{B}_{2}\right)$ is defined as follows. $\mathcal{B}_{1}$ samples a random string $s^{*} \in\{0,1\}^{\lambda}$ and outputs the set $\left\{s^{*}\right\}$ as the punctued set and the point $s^{*}$ as the state.

Next, $\mathcal{B}_{2}$ gets as input a punctured PRF key $K^{\prime} \triangleq K_{\left\{s^{*}\right\}}$ at the point $s^{*}$, a value $y$ (which is either $\operatorname{PRF}_{K}\left(s^{*}\right)$ or a random string), and the point $s^{*}$ as the state information. Next, $\mathcal{B}_{2}$ chooses a master key msk $\leftarrow$ FE.Setup $\left(1^{\lambda}\right)$ and emulates the execution of $\mathcal{A}_{1}$ on input $1^{\lambda}$ by simulating the encryption oracle and the key generation oracle as follows: When $\mathcal{A}_{1}$ requests an encryption of $x \in \mathcal{X}_{\lambda}, \mathcal{B}_{2}$ samples $s \in\{0,1\}^{\lambda}$, computes FE.Enc(msk, $\left.(x, \perp, s, \perp)\right)$ and returns the output to $\mathcal{A}_{1}$. When $\mathcal{A}_{1}$ requests the $j^{\text {th }}$ functional key for $f_{j} \in \mathcal{F}, \mathcal{B}_{2}$ samples a PRF key $K_{j} \leftarrow \operatorname{PRF}$.Gen $\left(1^{\lambda}\right)$, computes FE.KG(msk, Right $\left.f_{j}, K_{j}\right)$ if $j<i$, computes FE.KG(msk, PuncOutputZ $\left.f_{f_{j}, K^{\prime}, s^{*}}\right)$ if $j=i$ and computes FE.KG(msk, Left $\left.f_{j}, K_{j}\right)$ if $j>i$, and returns the output to $\mathcal{A}_{1}$. Finally, $\mathcal{A}_{1}$ outputs the challenge $\left(x_{0}^{*}, x_{1}^{*}\right.$, state).

Then, $\mathcal{B}_{2}$ chooses a random bit $b$, computes $c^{*} \leftarrow$ FE.Enc $\left(\right.$ msk, $\left.\left(x_{b}^{*}, x_{1}^{*}, s^{*}, f_{i}\left(x_{b}^{*}, y\right)\right)\right)$ and emulates the execution of $\mathcal{A}_{2}$ on input ( $c^{*}$, state) by simulating the encryption oracle as before and simulating 
the key generation oracle as follows: When $\mathcal{A}_{2}$ requests a functional key for $f \in \mathcal{F}, \mathcal{B}_{2}$ computes FE.KG(msk, PuncOutputY $\left.Y_{f, K^{\prime}, f\left(x_{1}^{*}\right), s^{*}}\right)$ and returns the outputs to $\mathcal{A}_{2}$. Finally, when $\mathcal{A}_{2}$ outputs the bit $b^{\prime}, \mathcal{B}_{2}$ outputs 1 if $b^{\prime}=b$, and otherwise it outputs 0 .

Note that when $y$ is obtained from as a PRF evaluation then $\mathcal{A}$ 's view is identical to its view in the experiment $\mathcal{H}^{(6, i)}$, and when $y$ is obtained from as a random string then $\mathcal{A}$ 's view is identical to its view in the experiment $\mathcal{H}^{(7, i)}$. Therefore,

$$
\left|\operatorname{Pr}\left[\mathcal{H}^{(6, i)}(\lambda)=1\right]-\operatorname{Pr}\left[\mathcal{H}^{(7, i)}(\lambda)=1\right]\right|=\operatorname{Adv}_{\text {puPRF }, \mathcal{B}^{(6, i) \rightarrow(7, i)}}(\lambda) .
$$

Proof of Claim 4.19. The adversary $\mathcal{B}^{(7, i) \rightarrow(8, i)}=\left(\mathcal{B}_{1}, \mathcal{B}_{2}\right)$ is defined as follows. First, $\mathcal{B}_{1}$ chooses a master key msk $\leftarrow$ FE.Setup $\left(1^{\lambda}\right)$ and emulates the execution of $\mathcal{A}_{1}$ on input $1^{\lambda}$ by simulating the encryption oracle and the key generation oracle as follows: When $\mathcal{A}_{1}$ requests an encryption of $x \in$ $\mathcal{X}_{\lambda}, \mathcal{B}_{1}$ samples $s \in\{0,1\}^{\lambda}$, computes FE.Enc $(\mathrm{msk},(x, \perp, s, \perp))$ and returns the output to $\mathcal{A}_{1}$. When $\mathcal{A}_{1}$ requests the $j^{\text {th }}$ functional key for $f_{j} \in \mathcal{F}, \mathcal{B}_{2}$ samples a PRF key $K_{j} \leftarrow \operatorname{PRF}$.Gen $\left(1^{\lambda}\right)$, samples $s^{*} \leftarrow\{0,1\}^{\lambda}$, computes FE.KG(msk, Right $\left.f_{j}, K_{j}\right)$ if $j<i$, computes FE.KG(msk, PuncOutputZ $\left.f_{j}, K^{\prime}, s^{*}\right)$ if $j=i$ and computes FE.KG(msk, Left $\left.f_{j}, K_{j}\right)$ if $j>i$, and returns the output to $\mathcal{A}_{1}$. When, $\mathcal{A}_{1}$ outputs the challenge $\left(x_{0}^{*}, x_{1}^{*}\right.$, state $), \mathcal{B}_{1}$ chooses a random bit $b$, and outputs $\left(f_{i}, x_{b}^{*}, x_{1}^{*}\right)$ and its entire memory and internal randomness as the state information.

Next, $\mathcal{B}_{2}$ runs on input $y$ (which is either a uniform sample from $f\left(x_{b}^{*}\right)$ or from $f\left(x_{1}^{*}\right)$ ), computes $c^{*} \leftarrow$ FE.Enc $\left(m s k,\left(x_{b}^{*}, x_{1}^{*}, s^{*}, y\right)\right)$, emulates the execution of $\mathcal{A}_{2}$ on input ( $c^{*}$, state) by simulating the encryption algorithm as before and simulating the key generation oracle as follows: When $\mathcal{A}_{2}$ requests a functional key for $f \in \mathcal{F}, \mathcal{B}_{2}$ computes FE.KG(msk, PuncOutputY $\left.Y_{f, K^{\prime}, f\left(x_{1}^{*}\right), s^{*}}\right)$ and returns the outputs to $\mathcal{A}_{2}$. Finally, when $\mathcal{A}_{2}$ outputs the bit $b^{\prime}, \mathcal{B}_{2}$ outputs 1 if $b^{\prime}=b$, and otherwise it outputs 0 .

Note that when $y$ is sampled from $f\left(x_{b}^{*}\right)$ then $\mathcal{A}$ 's view is identical to its view in the experiment $\mathcal{H}^{(7, i)}$, and when $y$ is sampled from $f\left(x_{1}^{*}\right)$ then $\mathcal{A}$ 's view is identical to its view in the experiment $\mathcal{H}^{(8, i)}$. Therefore,

$$
\left|\operatorname{Pr}\left[\mathcal{H}^{(7, i)}(\lambda)=1\right]-\operatorname{Pr}\left[\mathcal{H}^{(8, i)}(\lambda)=1\right]\right|=\operatorname{Adv}_{\mathcal{F}, \mathcal{B}^{(7, i) \rightarrow(8, i)}}^{\mathrm{aADM}} \leq \Delta(\lambda) .
$$

